\title{
TIGIT and PD-1 impair tumor antigen-specific CD8+ T cells in melanoma patients
}

\author{
Joe-Marc Chauvin, ${ }^{1}$ Ornella Pagliano, ${ }^{1}$ Julien Fourcade, ${ }^{1}$ Zhaojun Sun, ${ }^{1}$ Hong Wang, ${ }^{2}$ Cindy Sander, ${ }^{1}$ John M. Kirkwood, ${ }^{1}$ \\ Tseng-hui Timothy Chen, ${ }^{3}$ Mark Maurer, ${ }^{3}$ Alan J. Korman, ${ }^{3}$ and Hassane M. Zarour ${ }^{1,4}$ \\ 'Department of Medicine and Division of Hematology/Oncology and 'Department of Biostatistics, University of Pittsburgh, School of Medicine, Pittsburgh, Pennsylvania, USA. ${ }^{3}$ Biologics Discovery California, \\ Bristol-Myers Squibb, Redwood City, California, USA. ${ }^{4}$ Department of Immunology, University of Pittsburgh, School of Medicine, Pittsburgh, Pennsylvania, USA.
}

\begin{abstract}
T cell Ig and ITIM domain (TIGIT) is an inhibitory receptor expressed by activated T cells, Tregs, and NK cells. Here, we determined that TIGIT is upregulated on tumor antigen-specific (TA-specific) CD8+ $T$ cells and CD8 ${ }^{+}$tumor-infiltrating Iymphocytes (TILs) from patients with melanoma, and these TIGIT-expressing CD8 ${ }^{+} \mathrm{T}$ cells often coexpress the inhibitory receptor PD-1. Moreover, CD8+ TILs from patients exhibited downregulation of the costimulatory molecule CD226, which competes with TICIT for the same ligand, supporting a TICIT/CD226 imbalance in metastatic melanoma. TIGIT marked early T cell activation and was further upregulated by $T$ cells upon PD-1 blockade and in dysfunctional PD-1+TIM-3+ TA-specific CD8+ T cells. PD-1+TIGIT+, PD-1-TIGIT+, and PD-1+TICIT- CD8+ TILs had similar functional capacities ex vivo, suggesting that TICIT alone, or together with PD-1, is not indicative of T cell dysfunction. However, in the presence of TIGIT ligand-expressing cells, TICIT and PD-1 blockade additively increased proliferation, cytokine production, and degranulation of both TA-specific CD8 ${ }^{+}$T cells and CD8+ ${ }^{+}$TILs. Collectively, our results show that TIGIT and PD-1 regulate the expansion and function of TA-specific CD8 ${ }^{+}$ T cells and CD8+ TILs in melanoma patients and suggest that dual TICIT and PD-1 blockade should be further explored to elicit potent antitumor $\mathrm{CD8}^{+} \mathrm{T}$ cell responses in patients with advanced melanoma.
\end{abstract}

\section{Introduction}

$\mathrm{T}$ cells recognize tumor antigens expressed by melanoma cells but often fail to promote tumor regression in humans (1). There is now ample evidence that tumor antigen-specific (TA-specific) $\mathrm{CD}^{+} \mathrm{T}$ cells become dysfunctional and exhausted upon chronic antigen exposure, losing their capacity to proliferate, produce cytokines, and lyse tumor cells $(2,3)$. Dysfunctional TA-specific $\mathrm{CD}^{+} \mathrm{T}$ cells upregulate a number of inhibitory receptors including PD-1, TIM-3, and BTLA, which bind to their respective ligands expressed by antigen-presenting cells (APCs) and tumor cells, impeding $\mathrm{T}$ cell survival and functions in the tumor microenvironment (TME) (4-8). Blocking Abs targeting these inhibitory receptors successfully improve $\mathrm{T}$ cell responses in vitro and promote tumor regression in vivo in animals $(5-7,9,10)$. Immune checkpoint blockade with anti-PD-1 Abs has provided persistent clinical benefits for approximately $30 \%$ to $40 \%$ of patients with advanced melanoma in multiple clinical trials $(11,12)$. In addition, dual PD-1 and CTLA-4 blockade appears to further improve clinical outcome in patients (13). It is therefore expected that targeting multiple inhibitory pathways in the TME will prove useful for the majority of patients with advanced cancers, including melanoma.

$\mathrm{T}$ cell Ig and ITIM domain (TIGIT) is an inhibitory receptor that is expressed by activated T cells, Tregs, and NK cells and binds the adhesion molecules CD155 (Necl-5, also known as PVR) and CD112 (nectin-2, also known as PRR2 or PVRL2) with high

Conflict of interest: The authors have declared that no conflict of interest exists Submitted: December 10, 2014; Accepted: March 5, 2015

Reference information: J Clin Invest. 2015;125(5):2046-2058. doi:10.1172/JCI80445. and low affinity, respectively (14-17). CD155 and CD112 also bind to other ligands including the costimulatory counterpart to TIGIT, CD226 (DNAM-1), which associates with LFA-1 to positively regulate T cell responses $(18,19)$, and CD96 (20). CD155 and CD112 play a role in T cell- and NK cell-mediated cytotoxicity against tumors $(21,22)$. CD155 is expressed by neural tissues (23), endothelial cells, epithelial cells, platelets, $\mathrm{CD} 14^{+}$cells, and DCs, as well as by activated T cells and TLR-activated B cells (16, 23-27). CD112 is expressed by endothelial cells, hematopoietic cells, and immune cells including activated T cells and B cells, CD14 ${ }^{+}$cells, and DCs (28-30). Notably, CD155 and CD112 are also expressed by various human tumors, including melanoma (31-34).

Initial studies suggested that TIGIT exerts its immunosuppressive effects by enhancing IL-10 production by DCs through $\mathrm{CD} 155$, impeding $\mathrm{CD} 4^{+} \mathrm{T}$ cell proliferation and function (16). However, it was later demonstrated that TIGIT also exerts CD $4^{+}$ T cell-intrinsic inhibitory effects via recruitment of SHP phosphatases that suppress cytokine production and proliferation $(35,36)$ and competes with CD226 for PVR binding (37). The TIGIT locus is demethylated in Tregs and may potentially bind to FOXP3 (38). TIGIT $^{+}$Tregs are highly activated, secrete the soluble effector molecule fibrinogen-like protein 2, and selectively inhibit Th1 and Th17 responses (39).

Most recently, TIGIT expression by $\mathrm{CD}^{+}$tumor-infiltrating lymphocytes (TILs) has been reported using gene expression analyses in a number of mouse and human solid tumors including lung, colon, breast, uterine, and renal cancers. Elevated TIGIT expression appears to correlate with CD8 and PD-1 expression. TIGIT expression on $\mathrm{CD}^{+}$TILs was observed in 
mouse tumors and in 3 human tumor samples, including nonsmall-cell lung and colon cancers (40). Interestingly, TIGIT blockade synergized with PD-L1 blockade to enhance CD8 ${ }^{+}$TIL functions in mice and promoted the rejection of transplanted tumors, while single-agent blockade had no effect (40). Whether TIGIT is upregulated by TA-specific $\mathrm{CD}^{+} \mathrm{T}$ cells in the periphery and at tumor sites in patients with advanced melanoma remains unknown. In addition, its role in regulating the expansion and function of TA-specific $\mathrm{CD}^{+} \mathrm{T}$ cells in melanoma patients has not yet been investigated. Here, we show that TIGIT is upregulated and coexpressed with PD-1 on the majority of circulating TA-specific CD8 ${ }^{+} \mathrm{T}$ cells directed against the cancer germline antigen NY-ESO- 1 and on the majority of CD8 ${ }^{+}$TILs isolated from metastatic melanoma. CD8 ${ }^{+}$TILs downregulated CD226, supporting an imbalance of TIGIT/CD226 expression in metastatic melanoma. The TIGIT ligands CD155 and CD112 were upregulated by the majority of APCs and melanoma cells in the TME. TIGIT blockade added to PD-1 blockade to increase the expansion and functions of circulating TA-specific CD8 ${ }^{+} \mathrm{T}$ cells and $C D 8^{+}$TILs. Altogether, our findings support the use of TIGIT blockade in combination with PD-1 blockade to enhance CD8 ${ }^{+}$ $T$ cell responses to melanoma and improve the clinical efficacy of PD-1 blockade for patients with advanced melanoma.

\section{Results}

TIGIT and PD-1 are coexpressed on NY-ESO-1-specific CD8 ${ }^{+}$ $T$ cells. Using HLA-A2 (A2) tetramers, TIGIT expression on the surface of NY-ESO-1-, influenza- (Flu), and CMV-specific

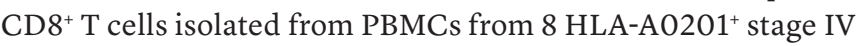
melanoma patients was assessed by flow cytometry ex vivo. In melanoma patients, the frequencies of TIGIT $^{+}$cells among A2/ NY-ESO-1-specific CD8 ${ }^{+} \mathrm{T}$ cells (mean frequency, 90.7\% $\pm \mathrm{SD}$ 4.1\%) were significantly higher than those among Flu-specific, CMV-specific, effector (CD45RA $\left.{ }^{+} \mathrm{CCR}^{-}\right)$, and effector memory (CD45RO ${ }^{+} \mathrm{CCR7}^{-}$) $\mathrm{CD}^{+} \mathrm{T}$ cells $(40.1 \% \pm 12.2 \%, 45.4 \% \pm 26.7 \%$, $57.7 \% \pm 20.33 \%$, and $15.9 \% \pm 15.9 \%$, respectively; Figure $1, \mathrm{~A}$ and B). Similar results were observed in terms of mean fluorescence intensity (MFI) (Figure 1B).

We next assessed the coexpression of PD-1 and TIGIT ex vivo on NY-ESO-1-, Flu-, and CMV-specific $\mathrm{CD}^{+}{ }^{+} \mathrm{T}$ cells. The large majority of NY-ESO-1-specific CD8 ${ }^{+} \mathrm{T}$ cells coexpressed TIGIT and PD-1, with mean frequencies of TIGIT $^{+} \mathrm{PD}-1^{+}$NY-ESO-1-specific $\mathrm{CD}^{+} \mathrm{T}$ cells $(83 \% \pm \mathrm{SD} 7.8 \%)$ being significantly higher than those of TIGIT $^{+}$PD-1- ${ }^{-}$, TIGIT-PD-1+ ${ }^{+}$, and TIGIT $^{-}$PD- $1^{-}$cells $(6.8 \%$ $\pm 3.3 \%, 5 \% \pm 2.8 \%$ and $5.3 \% \pm 3.9 \%$ respectively; Figure $1, \mathrm{C}$ and D). TIGIT and PD-1 coexpression on NY-ESO-1-specific CD8 ${ }^{+}$ $\mathrm{T}$ cells was positively correlated in terms of frequencies and MFI $(r=0.77, P=0.025$ and $r=0.092, P=0.0012$, respectively; Supplemental Figure 1, A and B; supplemental material available online with this article; doi:10.1172/JCI80445DS1). In sharp contrast to NY-ESO-1-specific CD8 ${ }^{+} \mathrm{T}$ cells, Flu- and CMV-specific $\mathrm{CD}^{+} \mathrm{T}$ cells, as well as effector and effector memory tet ${ }^{-} \mathrm{CD} 8^{+}$ $\mathrm{T}$ cells, were predominantly $\mathrm{TIGIT}^{-} \mathrm{PD}-\mathrm{1}^{-}$(mean frequency of $56 \% \pm$ SD $17 \%, 50.2 \% \pm 23.7 \%, 37.6 \% \pm 17.7 \%$, and $51.7 \% \pm 13.1 \%$, respectively), while $\mathrm{TIGIT}^{+} \mathrm{PD}-1^{+}$cells $(5.4 \% \pm 3.9 \%, 7.4 \% \pm 7.7 \%$, and $14.9 \% \pm 7.5 \%$, respectively) and TIGIT ${ }^{-}$PD $-1^{+}$cells $(9.2 \% \pm$ $8.1 \%, 4.2 \% \pm 5.5 \%, 4.7 \% \pm 4.3 \%$, and $7.5 \% \pm 6.3 \%$, respectively) represented small subsets of cells. In contrast to NY-ESO-1-specific CD8 ${ }^{+} \mathrm{T}$ cells, TIGIT and PD-1 were rarely coexpressed by Flu- or CMV-specific $\mathrm{CD}^{+} \mathrm{T}$ cells (Figure $1, \mathrm{C}$ and D).

We have also evaluated TIGIT expression on different subsets of mononuclear cells including $\mathrm{CD}^{+} \mathrm{T}$ cells, $\mathrm{CD} 4^{+} \mathrm{T}$ cells, NK cells $\left(\mathrm{CD}^{+} 6^{+}\right)$, B cells $\left(\mathrm{CD} 19^{+}\right)$, monocytes $\left(\mathrm{CD} 14^{+}\right)$, and myeloid DCs (mDCs) $\left(\mathrm{CD} 11 \mathrm{c}^{+}\right)$isolated from PBMCs from melanoma patients and healthy donors. TIGIT was expressed on subsets of $\mathrm{CD}^{+} \mathrm{T}$ cells, $\mathrm{CD} 4^{+} \mathrm{T}$ cells, and NK cells, with no significant differences observed between melanoma patients and healthy donors (Supplemental Figure 1, C and D).

Collectively, our results demonstrate that TIGIT expression is upregulated on tumor-induced NY-ESO-1-specific CD8 ${ }^{+}$ $\mathrm{T}$ cells in patients with advanced melanoma. The vast majority of NY-ESO-1-specific CD8 ${ }^{+} \mathrm{T}$ cells coexpress TIGIT and PD-1, unlike Flu-specific, CMV-specific, tet ${ }^{-}$effector, or tet ${ }^{-}$effector memory $\mathrm{CD}^{+} \mathrm{T}$ cells in the same melanoma patients.

TIGIT ${ }^{+} P D-1^{+}$NY-ESO-1-specific CD $8^{+} T$ cells exhibit high levels of $T$ cell activation. We next assessed the differentiation and activation status of NY-ESO-1-specific and tet ${ }^{-} \mathrm{CD} 8^{+} \mathrm{T}$ cells according to TIGIT and/or PD-1 expression in patients with advanced melanoma. To this end, in 8 stage IV melanoma patients, we compared the percentages of $\mathrm{CD}^{+} \mathrm{T}$ cells, which express the following markers ex vivo: CCR7, CD45RA, HLA-DR, and CD38 among TIGIT-PD-1 ${ }^{-}$, TIGIT-PD-1 ${ }^{+}$, TIGIT $^{+}$PD-1 ${ }^{-}$, and TIGIT $^{+}$PD $-1^{+}$ tet $\mathrm{CD}^{+} \mathrm{T}$ cells. Because of the low frequencies of PD-1-TIGIT ${ }^{+}$, PD-1 ${ }^{+}$TIGIT $^{-}$, and PD-1-TIGIT ${ }^{-}$NY-ESO-1-specific CD8 ${ }^{+}$T cells, we compared the phenotype of $\mathrm{TIGIT}^{+} \mathrm{PD}-1^{+}$tet $^{+} \mathrm{CD} 8^{+} \mathrm{T}$ cells with

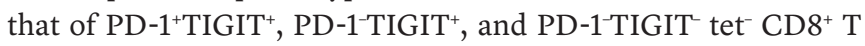
cells (Figure 2, A and B). The percentages of $\mathrm{HLA}-\mathrm{DR}^{+}$cells were higher among $\mathrm{PD}-1^{+} \mathrm{TIGIT}^{+}$tet ${ }^{-} \mathrm{CD}^{+} \mathrm{T}$ cells than among $\mathrm{PD}-1^{-}$ TIGIT $^{+}$and PD-1-TIGIT ${ }^{-}$tet $^{-} \mathrm{CD}^{+}{ }^{+} \mathrm{T}$ cells (mean percentage, $73.1 \% \pm$ SD $12.4 \%$ vs. $55.6 \% \pm 26.6 \%$ and $27.4 \% \pm 22.4 \%$, respectively). The frequencies of $\mathrm{CD}^{3} 8^{+}$cells were significantly higher among PD- $1^{+}$TIGIT $^{+}$NY-ESO-1-specific CD8 ${ }^{+} \mathrm{T}$ cells $(68.6 \%$ $\pm 18.5 \%)$ than among PD- $1^{-}$TIGIT $^{-}$tet $^{-} \mathrm{CD}^{+} \mathrm{T}$ cells $(22.4 \% \pm$ $14.4 \%)$ and $\mathrm{PD}-1^{-} \mathrm{TIGIT}^{+}$tet ${ }^{-} \mathrm{CD}{ }^{+} \mathrm{T}$ cells $(31.5 \% \pm 27.1 \%)$, but not

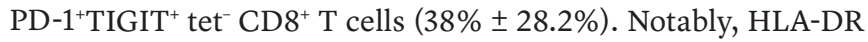
expression on $\mathrm{PD}-1^{+} \mathrm{TIGIT}^{+} \mathrm{NY}$-ESO-1-specific CD8 ${ }^{+} \mathrm{T}$ cells was significantly higher than on $\mathrm{PD}-1^{+} \mathrm{TIGIT}^{+}$tet $^{-} \mathrm{CD}{ }^{+}{ }^{+}$T cells $(83.1 \%$ $\pm 9.4 \%$ vs. $55.6 \% \pm 26.6 \%$, Figure $2 \mathrm{~B}$ ).

The percentages of $\mathrm{CCR}^{+}$and $\mathrm{CD}^{2} 5 \mathrm{RA}^{+} \mathrm{TIGIT}^{+} \mathrm{PD}-1^{+}$tet $^{-}$ $\mathrm{CD}^{+}{ }^{+} \mathrm{T}$ cells were lower than those of $\mathrm{TIGIT}^{+} \mathrm{PD}-1^{-}$and $\mathrm{TIGIT}^{-}$ PD-1 ${ }^{-}$tet $\mathrm{CD}^{+} \mathrm{T}$ cells $(20 \% \pm 14.6 \%$ vs. $31.9 \% \pm 27.5 \%$ and $49.8 \% \pm 29.8 \%$, respectively, for CCR7 and $36.1 \% \pm 14.8 \%$ vs. $57.8 \% \pm 23.4 \%$ and $56.8 \% \pm 16.9 \%$, respectively, for CD $45 \mathrm{RA}$ ). In addition, the percentages of CD45RA ${ }^{+}$cells were lower among TIGIT $^{+}$PD- ${ }^{+}$NY-ESO-1-specific $\mathrm{CD}^{+} \mathrm{T}$ cells than among TIGIT ${ }^{+} \mathrm{PD}-1^{+}$tet $^{-} \mathrm{CD} 8^{+}$T cells $(14.4 \% \pm 11.6 \%$ vs. $36.1 \% \pm 27.5 \%$; Figure $2 \mathrm{~B}$ ), while the percentages of $\mathrm{CCR} 7^{+}$cells were similar in these 2 cell subsets, supporting our finding that $\mathrm{TIGIT}^{+} \mathrm{PD}-1^{+}$ $\mathrm{CD} 8^{+} \mathrm{T}$ cells are effector memory cells.

We observed that TIGIT expression (MFI) was higher on dysfunctional PD- $1^{+}$TIM- $3^{+}$than on PD- $1^{+}$TIM- $3^{-}$NY-ESO-1-specific $\mathrm{CD}^{+} \mathrm{T}$ cells, suggesting that TIGIT expression is upregulated upon chronic antigen exposure (Figure 2C). To investigate further, we isolated NY-ESO-1-specific $\mathrm{CD}^{+} \mathrm{T}$ cells from the peripheral 
A

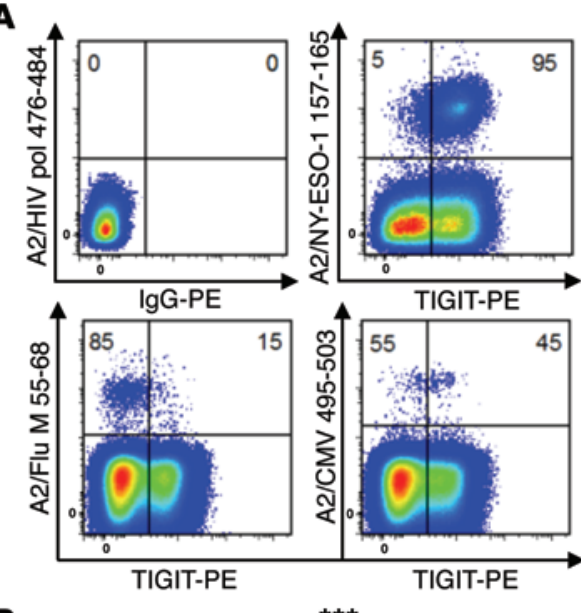

B
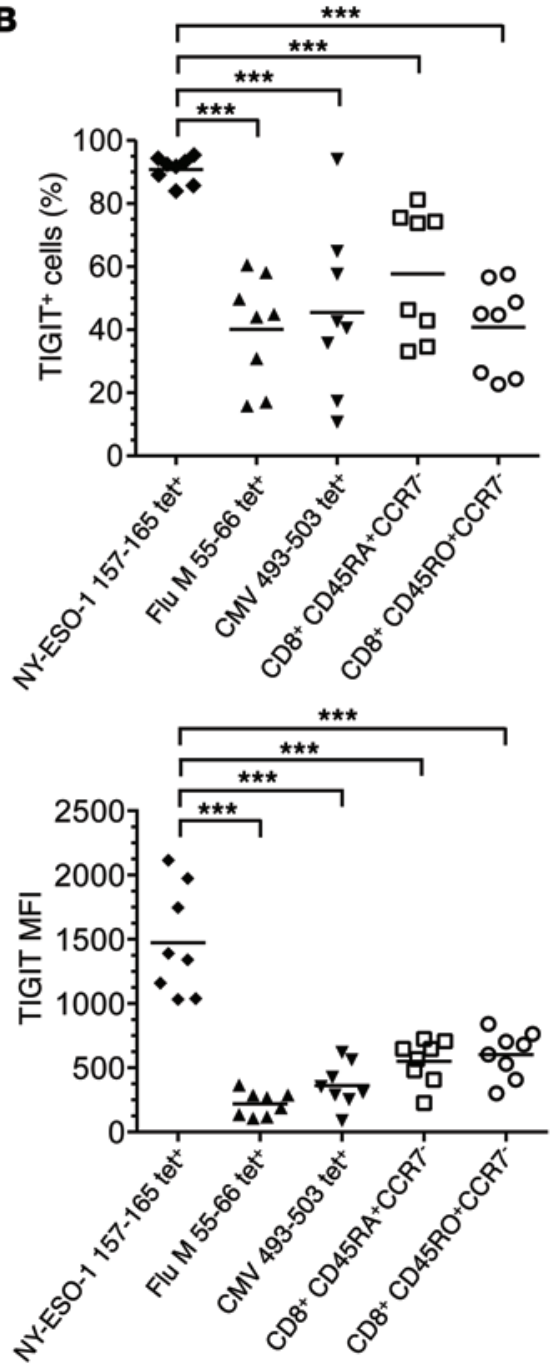

C

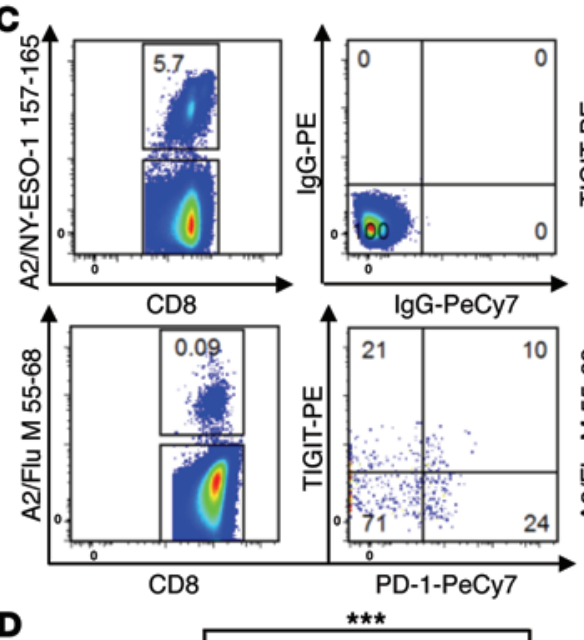

D
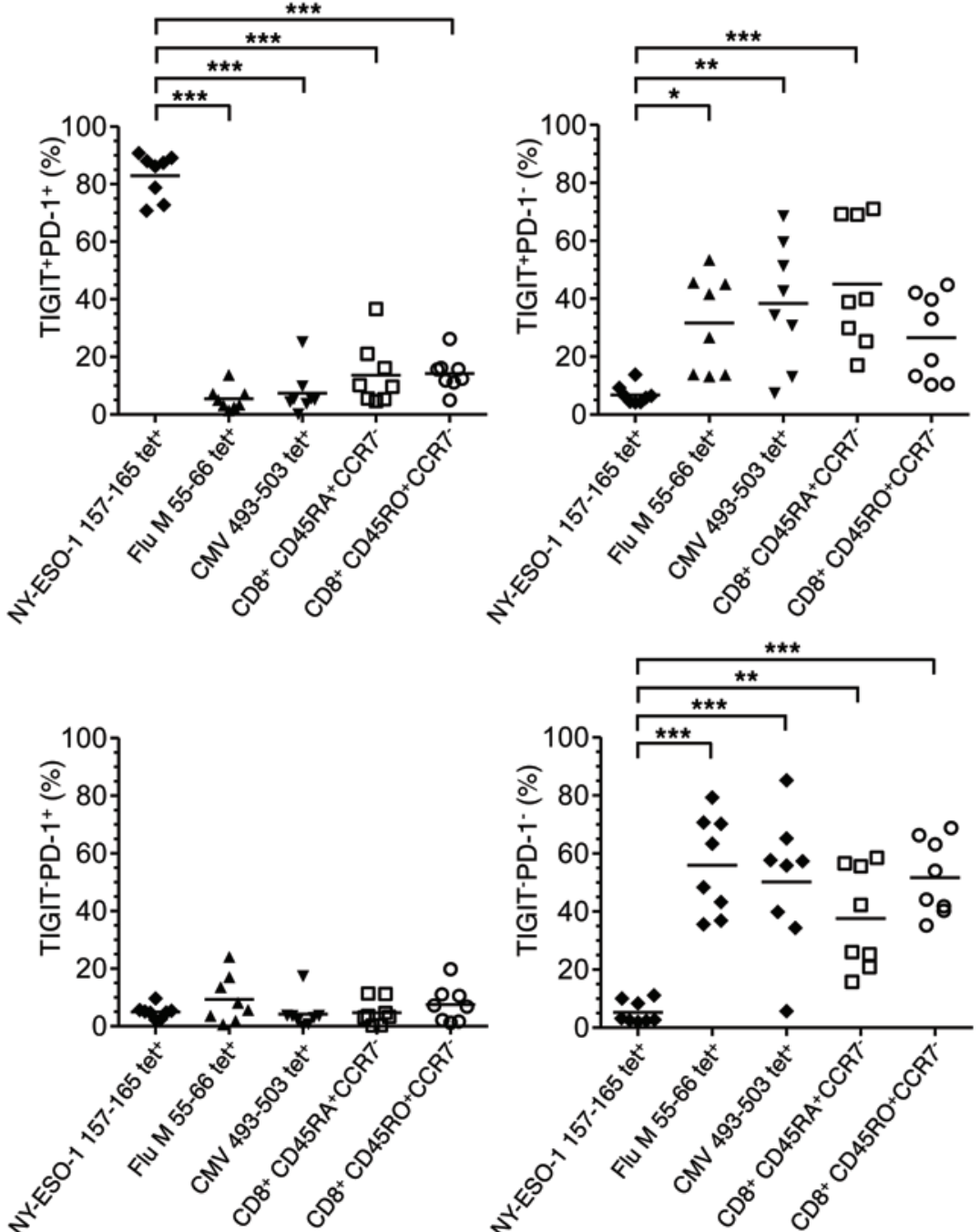

Figure 1. TIGIT is upregulated and coexpressed with PD-1 on NY-ESO-1-specific CD8+ $\mathbf{T}$ cells. (A) Representative dot plots for 1 melanoma patient showing ex vivo TIGIT expression on A2/NY-ESO-1 157-165, A2/Flu-M 58-66, and A2/CMV 495-503 tet ${ }^{+}$CD8 $^{+}$T cells. CD8 ${ }^{+}$T cells stained with A2/HIV pol 476-484 tetramers or PE-labeled IgG control mAbs were used to establish the threshold for identifying tet ${ }^{+}$and TIGIT+ $^{+}$cells, respectively. (B) Pooled data showing the percentage and MFI of TIGIT expression on NY-ESO-1-, Flu-, and CMV-specific CD8 ${ }^{+}$T cells as well as on total effector (CD45RA+CCR7-) and effector memory (CD45RO+CCR7-) CD8 ${ }^{+} T$ cells from melanoma patients $(n=8)$. $P$ values were obtained by repeated-measures ANOVA, followed by Tukey's multiple comparisons test. (C) Dot plots for 1 representative melanoma patient showing ex vivo TIGIT and PD-1 expression on A2/NY-ESO-1 157165, A2/Flu-M 58-66, and A2/CMV 495-503 tet ${ }^{+}$CD8 ${ }^{+}$T cells as well as on total tet- CD8 ${ }^{+}$T cells. (D) Pooled data showing the distribution of NY-ESO-1-, Flu-, and CMV-specific CD8 ${ }^{+}$T cells, as well as of total effector and effector memory CD8 ${ }^{+}$T cells according to TIGIT and PD- 1 expression in cells from melanoma patients $(n=8)$. $P$ values were obtained by Friedman's test, followed by Dunn's multiple comparisons test. Horizontal bars depict the mean percentage or MFI. ${ }^{*} P<0.05$; ${ }^{* *} P<0.01 ;{ }^{* *} P<0.001$. Data shown are representative of 3 independent experiments. 
A
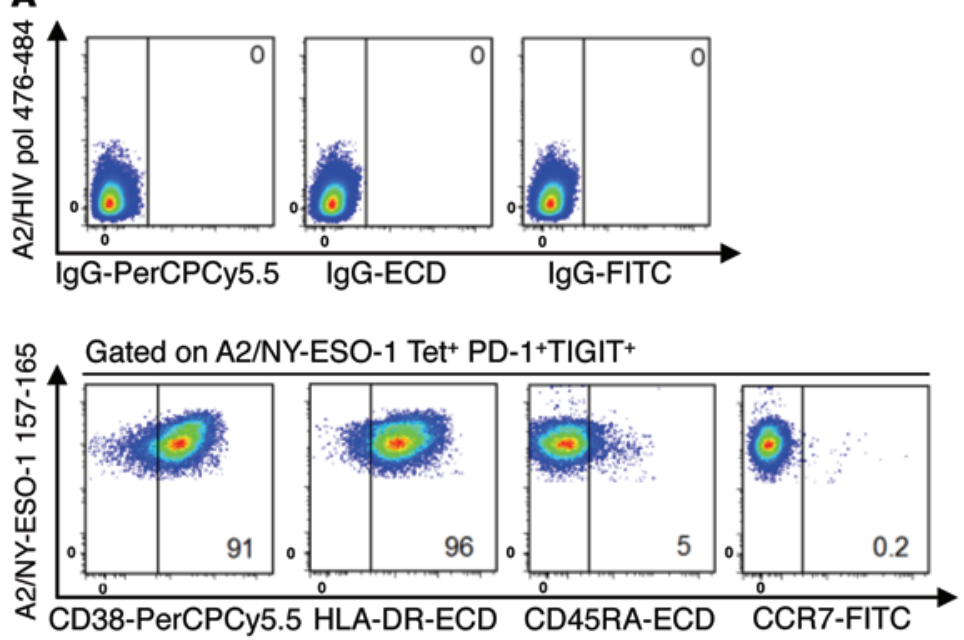

G Gated on Tet PD-1+TIGIT+

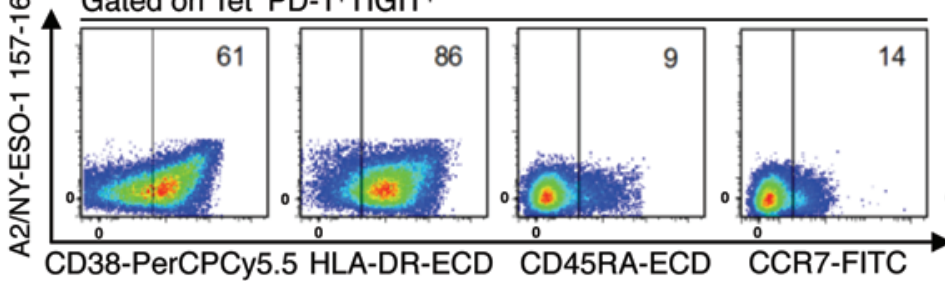

ㄴ. Gated on Tet PD-1-TIGIT+
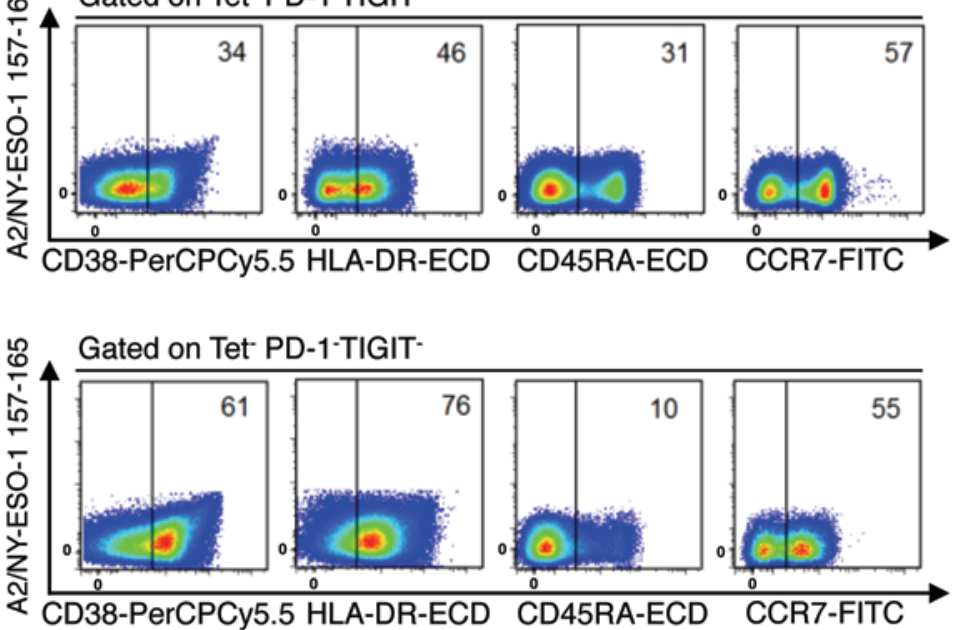

B
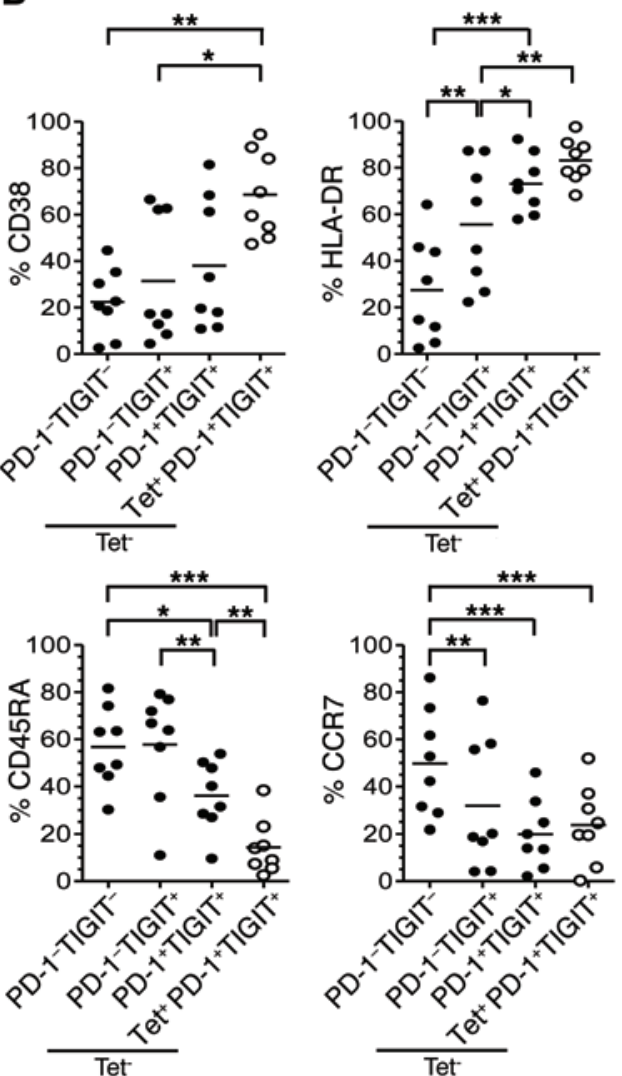

C

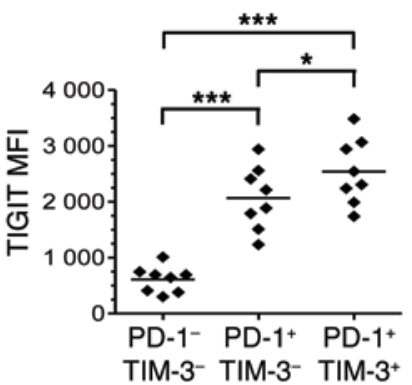

Figure 2. TIGIT'PD-1+ NY-ESO-1-specific CD8 ${ }^{+} \mathbf{T}$ cells exhibit an effector memory and activated T cell phenotype. (A and B) Representative dot plots for 1 melanoma patient (A) and summary data for 8 melanoma patients (B) indicating the frequencies (\%) of CD38, HLA-DR, CD45RA, and CCR7 expressed on A2/NY-ESO-1 157-165 tet ${ }^{+}$CD8 ${ }^{+}$T cells and tet ${ }^{-}$CD8 ${ }^{+}$T cells expressing TIGIT and/or PD-1. $P$ values were obtained by Friedman's test, followed by Dunn's multiple comparisons test (top left panel) and by repeated-measures ANOVA, followed by Tukey's multiple comparisons test (all other panels). (C) Ex vivo expression of TICIT (MFI) on A2/NY-ESO-1-specific CD8 ${ }^{+}$T cells according to PD-1 and TIM-3 coexpression. $P$ values were obtained by repeated-measures ANOVA, followed by Tukey's multiple comparisons test. Horizontal bars depict the mean percentage or MFI. ${ }^{*} P<0.05 ;{ }^{* *} P<0.01$; ${ }^{* *} P<0.001$.

blood of melanoma patients and assessed the expression of TIGIT over the 6 days of in vitro stimulation (IVS) with cognate or irrelevant peptide (Supplemental Figure 2, A and B). TIGIT expression (MFI) was increased after 24 hours of stimulation with cognate peptide but did not significantly increase thereafter. This differs considerably from PD-1 expression (5), which was not significantly increased until day 4 of stimulation.

Altogether, our findings demonstrate that TIGIT is an early activation marker expressed by TA-specific $\mathrm{CD} 8^{+} \mathrm{T}$ cells upon
TCR activation and is further upregulated by highly dysfunctional $\mathrm{PD}-1^{+} \mathrm{TIM}-3^{+} \mathrm{TA}$-specific $\mathrm{CD} 8^{+} \mathrm{T}$ cells in patients with advanced melanoma.

TIGIT blockade adds to PD-1 blockade to increase proliferation of NY-ESO-1-specific CD8 ${ }^{+} \mathrm{T}$ cells upon antigen stimulation. We next evaluated the effect of TIGIT blockade alone or in combination with PD-1 blockade on the proliferation of NY-ESO-1-specific $\mathrm{CD}^{+} \mathrm{T}$ cells upon cognate antigen stimulation. CFSE-labeled PBMCs from 9 patients with advanced melanoma were incu- 
bated for 6 days with cognate peptide in the presence of blocking mAbs against TIGIT (aTIGIT) and/or PD-1 (aPD-1) or of an irrelevant control $\mathrm{mAb}$ (Figure 3). As shown for 1 representative patient (Figure 3A) and for 9 patients, TIGIT or PD-1 blockade alone resulted in modest increases in the frequencies of $\mathrm{CFSE}^{\text {lo }}$ (Figure 3B) and total (Figure 3C) NY-ESO-1-specific CD8 ${ }^{+} \mathrm{T}$ cells as compared with frequencies observed in the presence of IgG control mAbs (fold-change of 1.4 and 1.3, respectively, for TIGIT blockade; Figure 3D and E). Dual TIGIT and PD-1 blockade further increased $\mathrm{CFSE}^{\text {lo }}$ and total NY-ESO-1-specific CD8 ${ }^{+} \mathrm{T}$ cell frequencies (Figure 3, B and C), resulting in a fold-change of 2.5 and 2.3, respectively, as compared with IgG control, aTIGIT, or aPD-1 mAbs alone (Figure 3, D and E).

Collectively, our findings show that upon stimulation with cognate antigen, dual TIGIT and PD-1 blockade stimulates stronger NY-ESO-1-specific CD8 ${ }^{+} \mathrm{T}$ cell proliferation than does either blockade alone.

TIGIT blockade alone or in combination with PD-1 increases the frequencies of cytokine-producing NY-ESO-1-specific CD8 ${ }^{+} \mathrm{T}$ cells upon stimulation with cognate antigen. We next assessed whether TIGIT blockade increases cytokine production by NY-ESO-1specific CD8 ${ }^{+} \mathrm{T}$ cells. To this end, we isolated PBMCs from 9 melanoma patients and stimulated the cells with NY-ESO-1 157165 peptide for 6 days in the presence of anti-TIGIT and/or antiPD-1-blocking or IgG control mAbs. On day 6, cells were restimulated with cognate peptide for a 6-hour intracellular cytokine assay prior to flow cytometry (Figure 4). As shown for 1 representative patient (Figure 4A) and for 9 patients (Figure 4B), TIGIT or PD-1 blockade alone increased the frequencies of IFN- $\gamma$ - and TNF-producing NY-ESO-1-specific CD8 ${ }^{+} \mathrm{T}$ cells as compared with IgG control mAbs, resulting in increases of 1.6- and 2.2fold, respectively, for TIGIT blockade and increases of 1.2- and 1.2-fold, respectively, for PD-1 blockade (Figure 4C). Dual blockade of TIGIT and PD-1 further increased the frequency of IFN- $\gamma-$ and TNF-producing NY-ESO-1-specific $\mathrm{CD}^{+} \mathrm{T}$ cells, resulting in increases of 2.7- and 4.3-fold, respectively (Figure 4C). Dual PD-1 and TIGIT blockade increased the frequency of IFN- $\gamma-$ and TNF-producing cells among total NY-ESO-1-specific CD8 ${ }^{+} \mathrm{T}$ cells as compared with IgG control mAbs, suggesting that it augmented NY-ESO-1-specific CD8 ${ }^{+} \mathrm{T}$ cell functions on a cell-by-cell basis (Supplemental Figure 3, A and B).

We next investigated the mechanisms supporting the inhibitory effects of TIGIT blockade on TA-specific $\mathrm{CD} 8^{+} \mathrm{T}$ cells and CD8 ${ }^{+}$TILs. A number of experimental studies have suggested that the TIGIT pathway impedes $\mathrm{T}$ cell function by promoting IL-10 production by APCs through CD155 engagement (16), exerting direct $\mathrm{T}$ cell-intrinsic effects, competing with CD226 for binding to the ligands, or disrupting CD226 homodimerization (35-37). We first investigated whether TIGIT binding to its ligands expressed by APCs impedes TA-specific $\mathrm{CD}^{+} \mathrm{T}$ cell function. To this end, PBMCs were isolated from patients with NY-ESO-1-expressing melanoma cells exhibiting spontaneous $\mathrm{CD}^{+} \mathrm{T}$ cell responses to NY-ESO-1 and incubated for 6 days in the presence of cognate peptide and TIGIT-Fc prior to measuring intracellular cytokine production by TA-specific $\mathrm{CD}^{+} \mathrm{T}$ cells (Supplemental Figure 4A). We observed no significant difference in cytokine production by NY-ESO-1-specific CD8 ${ }^{+} \mathrm{T}$ cells in the presence of TIGIT-Fc as compared with that in IgG control mAbs (Supplemental Figure 4A), suggesting that the binding of TIGITFc to its ligands expressed by APCs does not impede TA-specific $\mathrm{CD}^{+} \mathrm{T}$ cell function. We also evaluated IL-10 production in supernatants of PBMC cultures in the presence of cognate antigen and blocking mAbs against aTIGIT, aPD-1, or an irrelevant control mAb (Supplemental Figure 4B). PD-1 or TIGIT single blockade failed to decrease IL-10 production in the supernatant of PBMC cultures (Supplemental Figure 4B). These findings suggest that the immunoregulatory effects of the TIGIT pathway on TA-specific $\mathrm{CD}^{+} \mathrm{T}$ cells are not mediated by IL-10 production by APCs upon TIGIT ligation.

Altogether, our findings show that TIGIT blockade enhances the expansion of cytokine-producing NY-ESO-1-specific CD8 ${ }^{+} \mathrm{T}$ cells and adds to PD-1 blockade to further augment TA-specific $\mathrm{CD}^{+} \mathrm{T}$ cell cytokine production. They also suggest that the immunoregulatory effects of the TIGIT pathway on TA-specific CD ${ }^{+}$ $\mathrm{T}$ cells are not mediated through IL-10 production by APCs but rather through direct T cell-intrinsic effects.

PD-1 blockade increases TIGIT expression by NY-ESO-1-specific $C D 8^{+} T$ cells. Because TIGIT and PD-1 are both upregulated upon $\mathrm{T}$ cell activation, we next sought to assess whether circulating TA-specific CD8 ${ }^{+} \mathrm{T}$ cells upregulate TIGIT and PD-1 upon PD-1 and TIGIT blockade, respectively. To this end, PBMCs from melanoma patients were incubated with NY-ESO-1 157-165 peptide in the presence of anti-TIGIT- or anti-PD-1-blocking mAbs for 6 days prior to flow cytometric analysis of PD-1 and TIGIT, respectively (Figure 5). We observed that PD-1 blockade increased TIGIT expression by NY-ESO-1-specific CD8 ${ }^{+} \mathrm{T}$ cells in PBMCs from all tested patients compared with incubation with IgG control mAbs (MFI: $2,410 \pm 1,586$ vs. $1,802 \pm 1,481,1.5$-fold change of TIGIT expression as measured by MFI; Figure 5 , A and B). In sharp contrast, TIGIT blockade did not significantly augment PD-1 expression by NY-ESO-1-specific CD8 ${ }^{+} \mathrm{T}$ cells as compared with IgG control mAbs (Figure 5, A and B).

These results show that NY-ESO-1-specific $\mathrm{CD}^{+} \mathrm{T}$ cells upregulate TIGIT expression upon PD-1 blockade, whereas TIGIT blockade has no effect on PD-1 expression.

TIGIT ligands are highly expressed by tumor cells and APCs from melanoma patients. We next evaluated the ex vivo expression of the TIGIT ligands CD155 and CD112 on APCs and/or melanoma cells present in metastatic melanoma tumors (single-cell suspensions) and in PBMCs from healthy donors and patients with advanced melanoma (Figure 6). We detected low frequencies of monocytes (mean percentage, $4.5 \% \pm$ SD $7.8 \%$ ) and DCs $(0.3 \% \pm 0.4 \%)$ and high frequencies of CSPG $4^{+}$melanoma cells $(50.5 \% \pm 25.3 \%)$ (Figure 6A). CD155 and CD112 were expressed by a majority of the CSPG $4^{+}$melanoma cells (mean percentage, $73.4 \% \pm$ SD $25.7 \%$ and $68.9 \pm 30.3$, respectively). Strikingly, CD155 and CD112 expression levels on melanoma cells were higher than those of PD-L1, HVEM, and galectin-9 (mean percentage $45.2 \% \pm 27.2 \%, 8.3 \% \pm 11.8 \%$ and $5.1 \% \pm 6.1 \%$, respectively; Figure 6, B and C). Additionally, monocytes and DCs isolated from tumors expressed elevated CD155 when compared with those isolated from the peripheral blood of patients and healthy donors (mean percentage $64 \% \pm$ SD $23 \%$ vs. $23 \% \pm 25 \%$ and $19.9 \% \pm 18.5 \%$, respectively, for monocytes and $68 \% \pm 23.7 \%$ vs. $26.8 \pm 10.3 \%$ and $32.5 \% \pm 14.3 \%$, respectively, for 
A
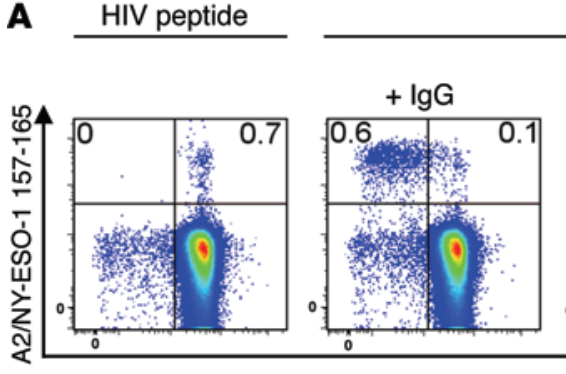

NY-ESO-1 peptide
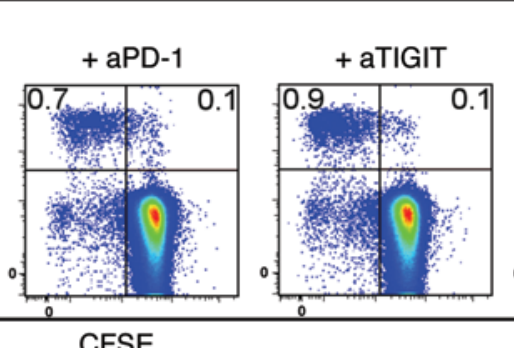

年

CFSE

B

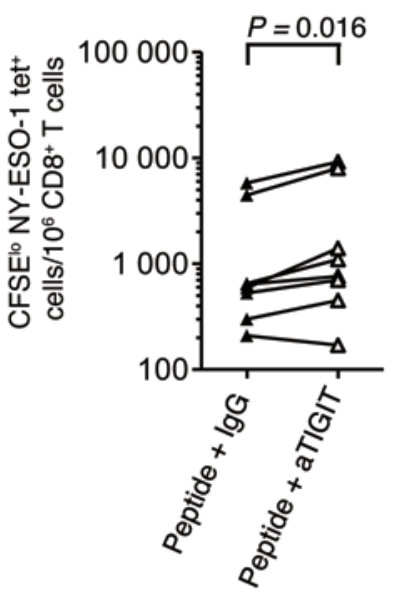

C

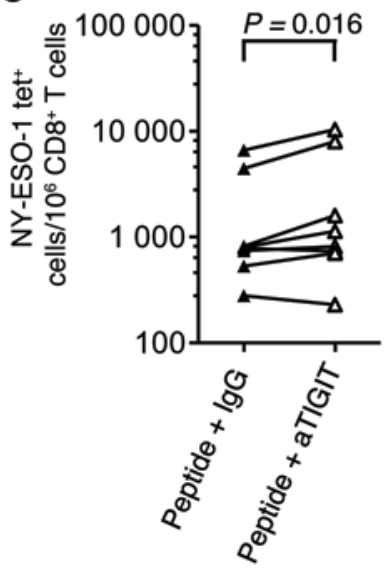

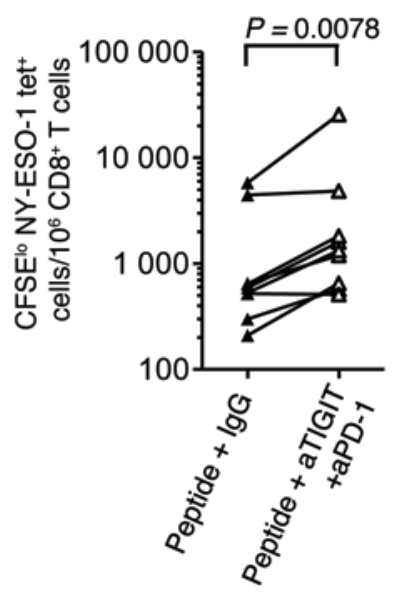

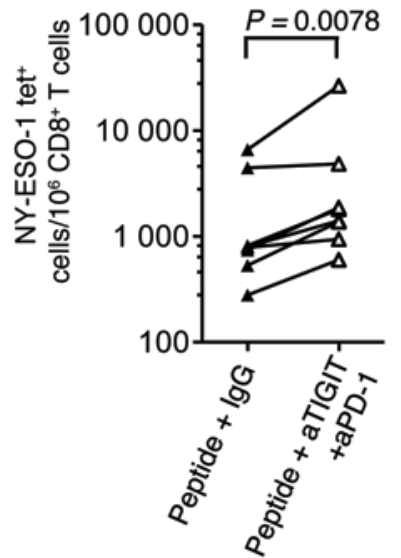

D

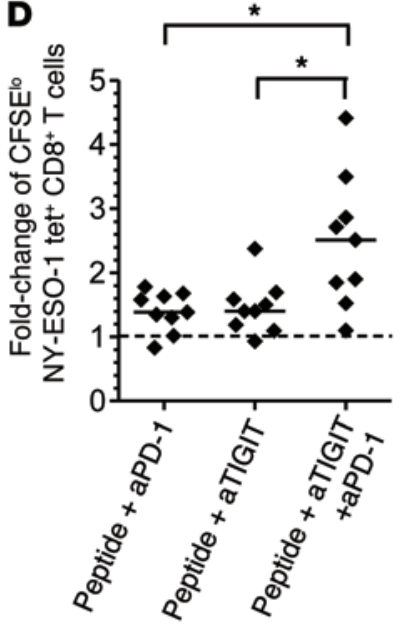

E

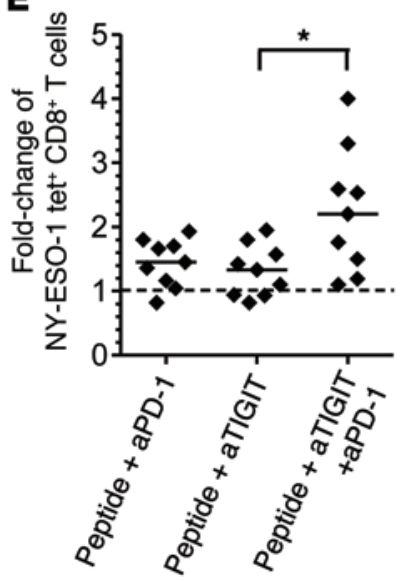

Figure 3. TICIT blockade alone or in combination with PD-1 blockade increases the frequency of proliferating and total NY-ESO-1specific CD8 $^{+} \mathbf{T}$ cells. CFSE-labeled PBMCs from melanoma patients were incubated in vitro for 6 days with NY-ESO-1 157-165 or HIV-pol 476-484 peptide and blocking mAbs against TIGIT (aTIGIT) and/ or PD-1 (aPD-1) or isotype control mAbs (IgG). (A) Representative flow cytometric analysis for $1 \mathrm{mel}$ anoma patient showing the percentages of $\mathrm{CFSE}^{10} \mathrm{~A} 2 / \mathrm{NY}$-ESO157-165 tet ${ }^{+} \mathrm{CD}^{+} \mathrm{T}$ cells among total CD8+ T cells. (B and C) Pooled data showing the variation in the numbers of CFSE $^{10}$ (B) and total (C) A2/NY-ESO-1 157-165 tet ${ }^{+}$cells per $10^{6} \mathrm{CD}^{+} \mathrm{T}$ cells $(n=9)$. $P$ values were obtained by 2-tailed, paired $t$ tests. ( $\mathbf{D}$ and $\mathbf{E}$ ) Fold-change of the frequencies of $\mathrm{CFSE}^{10}(\mathbf{D})$ and total (E) A2/NY-ESO-1 157-165 tet ${ }^{+}$ cells in the presence of the indicated blocking mAb $(n=9)$. $P$ values were obtained by repeated-measures ANOVA, followed by Tukey's multiple comparisons test. ${ }^{*} P<0.05$. Data shown are representative of 2 independent experiments performed in duplicate.
DCs; Figure 6, B and D). Similar observations were made in terms of MFI (925 \pm 240 vs. $304 \pm 138$ and $271 \pm 110$, respectively, for monocytes and $1,242 \pm 800$ vs. $246 \pm 63$ and $439 \pm 259$, respectively, for DCs). In addition, monocytes isolated from melanoma patients exhibited higher levels of CD112 expression in both melanoma tumors and PBMCs as compared with levels in peripheral monocytes from healthy donors (mean percentage, $82.2 \% \pm$ SD $11 \%$ and $82.1 \% \pm 16.7 \%$ vs. $42.7 \% \pm 30.9 \%$, respectively; Figure $6, \mathrm{~B}$ and D). Similar observations were made with regard to MFI $(1,376 \pm 1,171$ and $1,538 \pm 526$ vs. $252 \pm 96$, respectively).

The high expression levels of both TIGIT ligands by melanoma cells and melanoma-infiltrating APCs suggest that the TIGIT immunoregulatory pathway plays a critical role in regulating the expansion and functions of TA-specific $\mathrm{CD} 8^{+} \mathrm{T}$ cells within metastatic melanoma.
CD $8^{+}$TILs upregulate TIGIT and PD-1, and TIGIT blockade adds to PD-1 blockade to further increase the expansion of functional TILs after stimulation. We next investigated whether CD8 ${ }^{+} \mathrm{T}$ cells expressing TIGIT and PD-1 were present at the tumor site. In $\mathrm{CD}^{+}$TILs from metastatic melanoma patients (Figure 7, A and B, and Supplemental Figure 5A), we observed upregulation of TIGIT expression both in terms of frequency and MFI as compared with circulating $\mathrm{CD}^{+} \mathrm{T}$ cells from both melanoma patients and healthy donors (mean frequency, $67 \% \pm$ SD $17.9 \%$ vs. $43 \% \pm 13.5 \%$ and $30.8 \% \pm 18.5 \%$, respectively, and MFI, 1,317 \pm SD 748 vs. $493 \pm$ 352 and $676 \pm 766$, respectively; Figure 7A). Notably, the majority of $\mathrm{TIGIT}^{+} \mathrm{CD}^{+}$TILs coexpressed PD-1 (mean frequency of TIGIT $^{+}$PD $-1^{+}$CD $8^{+}$TILs, $47.6 \% \pm$ SD 19.7\%), while PD-1-TIGIT', PD-1 ${ }^{-}$TIGIT $^{+} \mathrm{CD}^{+}{ }^{+}$TILs, and PD- ${ }^{+}{ }^{+}$TIGIT $^{-} \mathrm{CD}^{+}$TILs represented much smaller $\mathrm{T}$ cell subsets $(25.7 \pm 18.5 \%, 19.8 \pm 10.9 \%$, and 6.8 
A

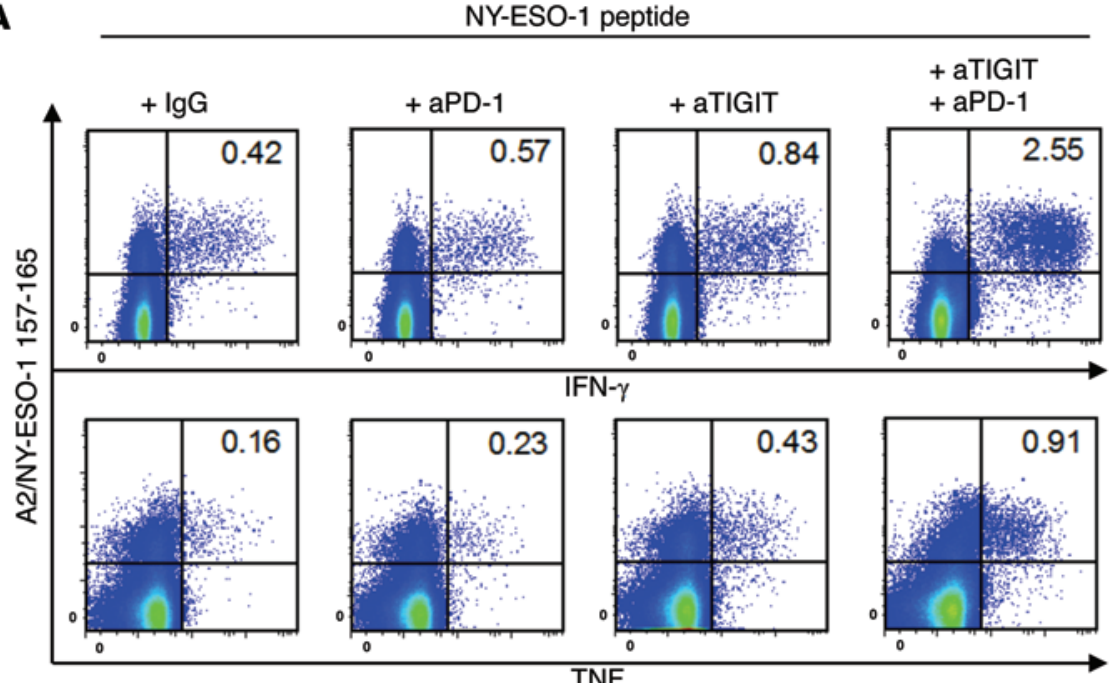

B
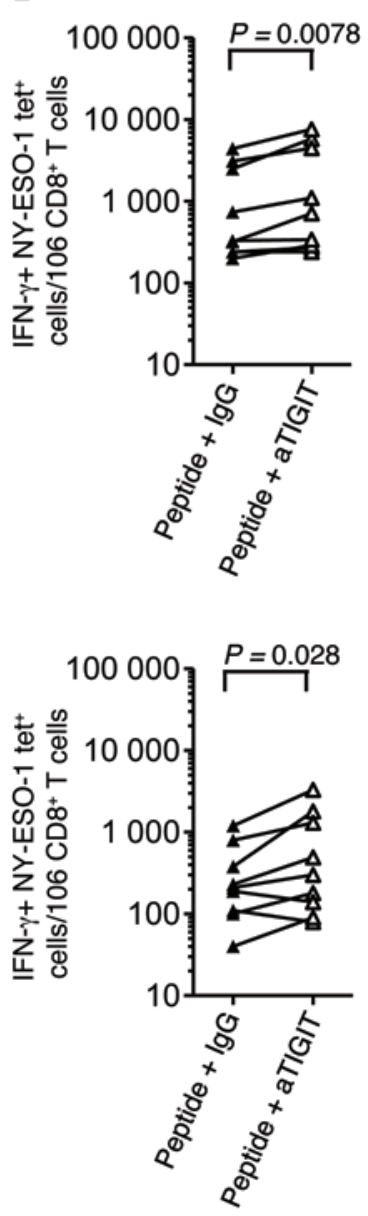

NY-ESO-1 peptide
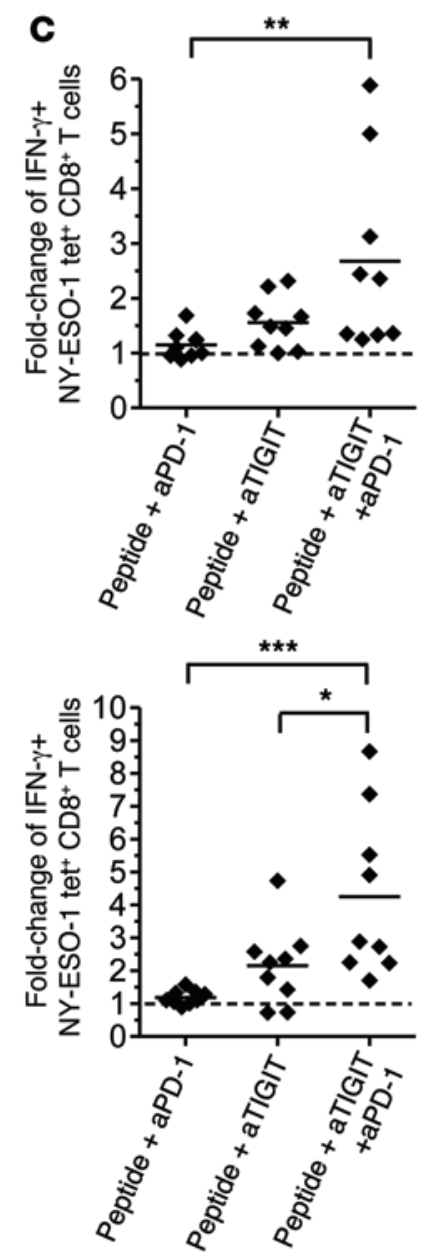

Figure 4. TIGIT blockade added to PD-1 blockade increases the frequencies of cytokine-producing NY-ESO-1-specific CD8 ${ }^{+}$ T cells. PBMCs from melanoma patients were incubated in vitro for 6 days with NY-ESO-1 157-165 peptide and blocking mAbs against TIGIT and/or PD-1 or isotype control mAbs (IgG) before evaluating intracellular cytokine production of A2/NY-ESO-1 157-165 tet ${ }^{+}$CD8 $T$ cells in response to the cognate peptide. (A and B) Representative flow cytometric analysis of cells from 1 melanoma patient $(\mathbf{A})$ and pooled data $(n=9)$ (B) showing the variation in numbers of IFN- $\gamma$ - and TNF-producing NY-ESO-1 157-165 tet ${ }^{+}$CD8 ${ }^{+} T$ cells per $10^{6} \mathrm{CD}^{+} \mathrm{T}$ cells. $P$ values were obtained by 2-tailed, paired $t$ tests. (C) Fold-change of frequencies of IFN- $\gamma$ - and TNF-producing A2/NY-ESO-1 157-165 tet ${ }^{+}$CD8 ${ }^{+} T$ cells after a 6 -day IVS with cognate antigen and the indicated mAb $(n=9)$. $P$ values were obtained by Friedman's test, followed by Dunn's multiple comparisons test (top panel) and by repeated -measures ANOVA (bottom panel), followed by Tukey's multiple comparisons test. ${ }^{*} P<0.05$; ${ }^{* *} P<0.01$; ${ }^{* * *} P<0.001$. Data shown are representative of 2 independent experiments performed in duplicate.

percentages of HLA-DR and CD38 (Supplemental Figure 5B), suggesting that these TILs are more differentiated and activated. Notably, we detected no significant difference between TIGIT-PD- $1^{+}$ and TIGIT $^{+} \mathrm{PD}-1^{-} \mathrm{CD}^{+}$TILs in terms of CD45RA, CCR7, CD38, HLA-DR, and CD57 expression (Supplemental Figure $5 B)$, suggesting that these 2 cell subsets exhibit similar states of differentiation and activation.

To investigate whether the upregulation of TIGIT expression by $\mathrm{CD} 8^{+}$ TILs correlates with $\mathrm{T}$ cell dysfunction, CD8 ${ }^{+}$TILs were stimulated with PMA and ionomycin in vitro prior to flow cytometric analysis of cytokine production (Figure 7C and Supplemental Figure $6 \mathrm{~A})$. We observed that PD-1+TIGIT ${ }^{+}$ and PD-1-TIGIT ${ }^{+} \mathrm{CD}^{+}$TILs exhibited IFN- $\gamma-$, TNF-, and IL-2-producing cells in frequencies similar to those of PD- $1^{+}$ TIGIT CD8 $8^{+}$TILs (Figure 7C). In sharp contrast, $\mathrm{PD}-1^{+} \mathrm{TIM}^{-} 3^{+} \mathrm{CD} 8^{+}$TILs pro-

$\pm 6.3 \%$, respectively; Figure 7, A and B). We also noticed that the majority of $\mathrm{CD} 8^{+}$TILs were $\mathrm{CD} 27^{+} \mathrm{CD} 28^{-}$and that $\mathrm{TIGIT}^{+} \mathrm{PD}-1^{+}$ $\mathrm{CD}^{+}{ }^{+}$TILs expressed more CD27 and CD57 than did TIGIT-PD-1, TIGIT-PD-1 $1^{+}$, or TIGIT ${ }^{+}$PD-1- ${ }^{-}$CD8 ${ }^{+}$TIL subsets (Supplemental Figure 5B). Similar to what we observed in the PBMCs from melanoma patients, and in sharp contrast to the TIGIT-PD-1-, TIGIT PD- $1^{+}$, and TIGIT $^{+}$PD- $1^{-}$CD8 ${ }^{+} \mathrm{T}$ cell subsets, the majority of $\mathrm{TIGIT}^{+} \mathrm{PD}-1^{+} \mathrm{CD}^{+}{ }^{+}$TILs were CD $45 \mathrm{RA}^{-} \mathrm{CCR}^{-}$and expressed high duced less TNF (mean percentage, $4.5 \% \pm$ SD 7.4\%) and IL-2 $(1.6 \% \pm 2.3 \%)$ than did PD-1 ${ }^{-T_{I G I T}}$, PD- $^{+}{ }^{+}$TIGIT $^{-}$, PD- $^{-}{ }^{-T_{G I T}}{ }^{+}$, or PD- $1^{+} \mathrm{TIGIT}^{+} \mathrm{CD}^{+}$TILs $(22.2 \% \pm 23.2,51.6 \% \pm 36.8 \%, 43.2 \% \pm$ $40.3 \%$, and $35.3 \% \pm 35.9 \%$, respectively, for TNF; $11.4 \% \pm 15.2 \%$, $27.1 \% \pm 24.7 \%, 18.2 \% \pm 17.5 \%$, and $14.7 \% \pm 14.7 \%$, respectively, for IL-2; Figure 7C).

We next evaluated the effects of TIGIT and PD-1 blockade on the capacity for proliferation and degranulation (CD107a expres- 
A
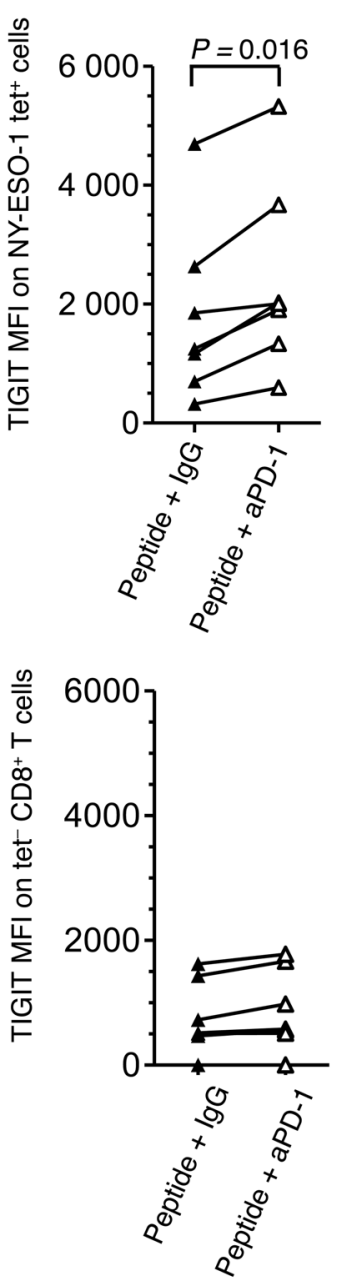

B
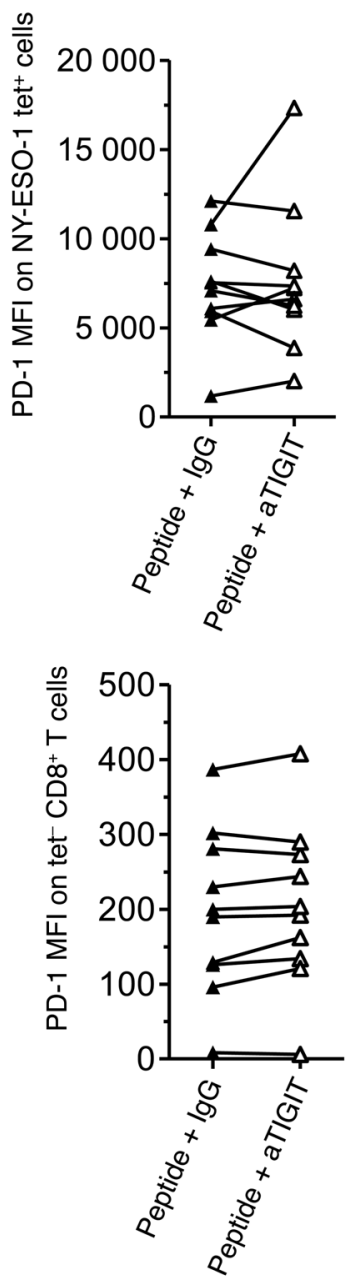
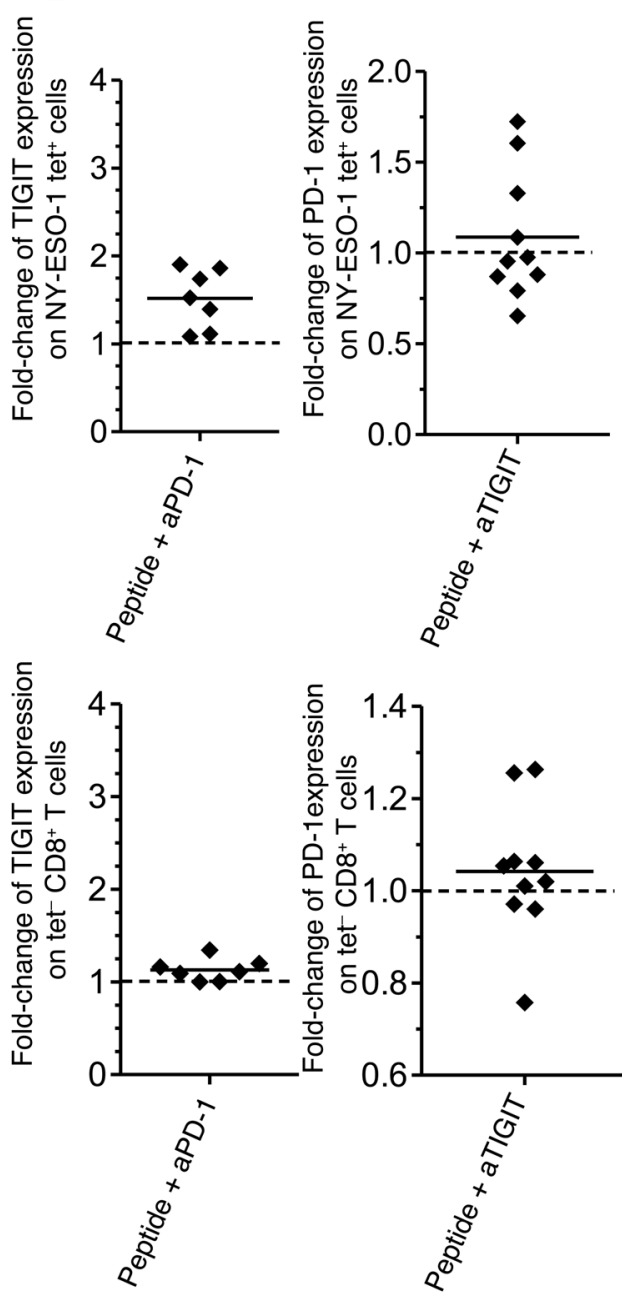

Figure 5. PD-1 blockade increases TIGIT expression by NY-ESO-1-specific CD8 ${ }^{+} \mathrm{T}$ cells. PBMCs from melanoma patients were incubated in vitro for 6 days with NY-ESO-1 157-165 peptide and blocking mAbs against TIGIT or PD- 1 or isotype control mAbs (IgG). (A and B) Pooled data for TIGIT $(n=7)$ and PD-1 $(n=10)$ expression as shown by MFI (A) and fold-change of expression (B) on A2/NY-ESO-1 157-165 tet ${ }^{+}$ CD8 ${ }^{+} \mathrm{T}$ cells and tet ${ }^{-} \mathrm{CD} 8^{+} \mathrm{T}$ cells after PD-1 and TIGIT blockade, respectively. $P=0.016$ by a 2 -tailed, paired $t$ test. Data are representative of 2 independent experiments performed in duplicate. sion) of CD8 ${ }^{+}$TILs upon TCR activation. CD8 ${ }^{+}$TILs were isolated from metastatic tumor single-cell suspensions from 7 patients with advanced melanoma, labeled with CFSE, and coincubated with autologous non-CD3 cells and anti-CD3 Ab in the presence of anti-TIGIT- and/or anti-PD-1-blocking mAbs (Figure 7D and Supplemental Figure 6B). We observed that TIGIT blockade increased the frequencies of proliferating $\left(\mathrm{CFSE}^{\mathrm{lo}}\right) \mathrm{CD}^{+} \mathrm{TILs}$ $(P=0.031)$ and CD107 $\mathrm{a}^{+} \mathrm{CD} 8^{+}$TILs in 5 of 6 patients as compared with IgG control mAbs. In addition, dual TIGIT and PD-1 blockade further increased the proliferation of $\mathrm{CD}^{+}$TILs as compared with IgG control mAbs $(P=0.016)$, as well as their degranulation as compared with IgG control mAbs $(P=0.031), P D-1$ blockade alone $(P=0.031)$, or TIGIT blockade alone $(P=0.031)$ (Figure 7D and Supplemental Figure 6B).

Collectively, our findings show that the majority of $\mathrm{CD}^{+}$TILs present in metastatic melanoma coexpress TIGIT and PD-1. Like circulating NY-ESO-1-specific CD8 ${ }^{+} \mathrm{T}$ cells from advanced melanoma patients, we found that $\mathrm{TIGIT}^{+} \mathrm{PD}-1^{+} \mathrm{CD} 8^{+}$TILs are highly activated effector memory $\mathrm{T}$ cells. While TIGIT expression by $\mathrm{CD}^{+}$TILs is not associated with $\mathrm{T}$ cell dysfunction in terms of cytokine production, our findings demonstrate that dual TIGIT and PD-1 blockade augments the proliferation and degranulation of $\mathrm{CD}^{+}$TILs isolated from metastatic melanoma.
$C D 8^{+}$TILs exhibit an imbalance of TIGIT/CD226 expression in metastatic melanoma. We next evaluated the expression of CD226 on total $\mathrm{CD}^{+} \mathrm{T}$ cells from healthy donors and patients with advanced melanoma, as well as on circulating NY-ESO-1-specific $\mathrm{CD}^{+} \mathrm{T}$ cells and CD8 ${ }^{+}$TILs from melanoma patients (Figure 8). CD226 was upregulated on circulating NY-ESO-1-specific CD8 ${ }^{+}$ $\mathrm{T}$ cells and total $\mathrm{CD}^{+} \mathrm{T}$ cells in PBMCs from healthy donors and melanoma patients in terms of both frequency (mean $99.8 \% \pm$ SD $0.4 \%, 89.35 \pm 6.7 \%$, and $91.3 \% \pm 4.1 \%$, respectively; Figure $8 \mathrm{~A}$ ) and MFI $(5,390 \pm 1,395,4,322 \pm 1,148$, and 4,193 $\pm 1,482$, respectively; Figure 8B). In sharp contrast, we detected lower CD226 ${ }^{+}$ $\mathrm{CD}^{+} \mathrm{T}$ cell frequencies in metastatic melanoma cells $(69.9 \% \pm$ $16.5 \%$; Figure $8 \mathrm{~A}$ ), and CD226 expression levels were strongly downregulated (MFI, 1,319 \pm SD 251; Figure 8B), suggesting an imbalance of TIGIT/CD226 expression in metastatic melanoma.

Collectively, our findings show an imbalance of TIGIT/CD226 expression by $\mathrm{CD}^{+}$TILs in metastatic melanoma that may enhance the negative immunoregulatory effects of TIGIT.

\section{Discussion}

In the present study, we report that TIGIT is upregulated by the majority of TA-specific $\mathrm{CD}^{+} \mathrm{T}$ cells in the periphery and within metastatic tumors of patients with advanced melanoma. In con- 
A $\quad$ B
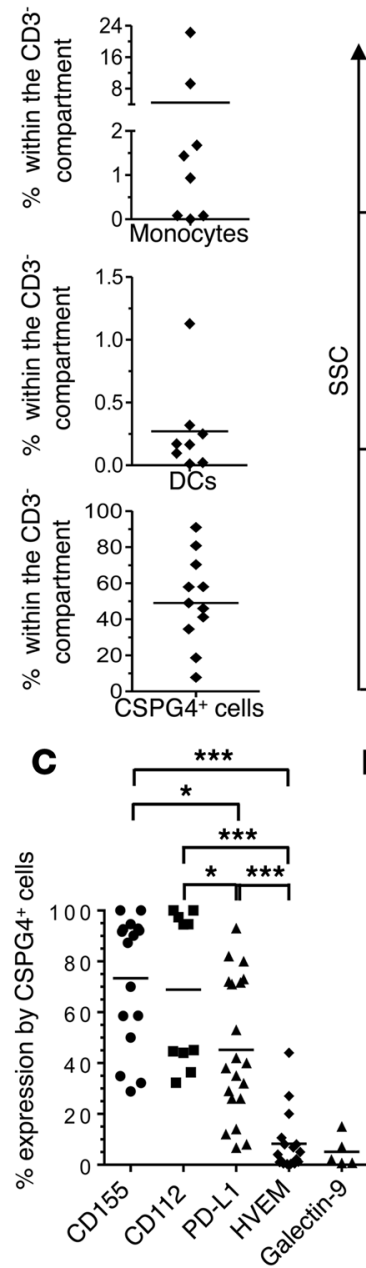

B

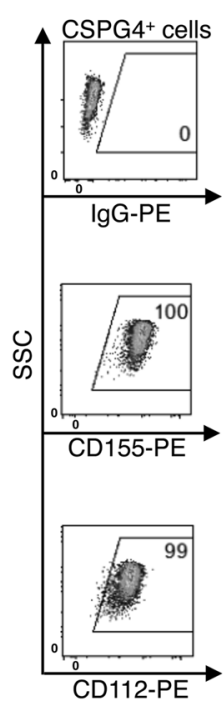

D
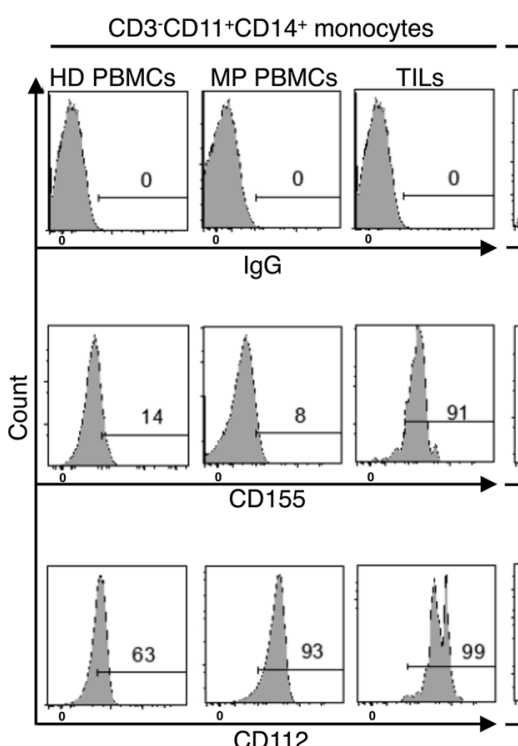

CD112
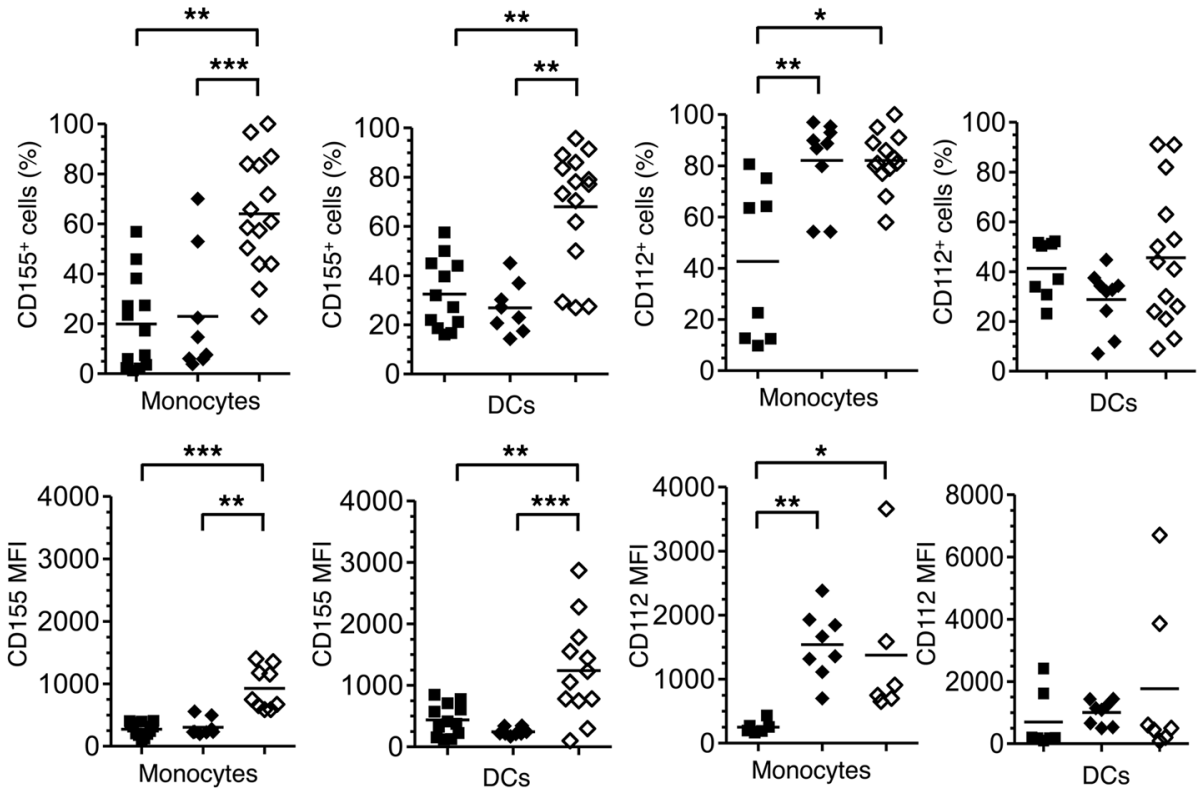

- Melanoma patients

- Healthy donors $\star \star$
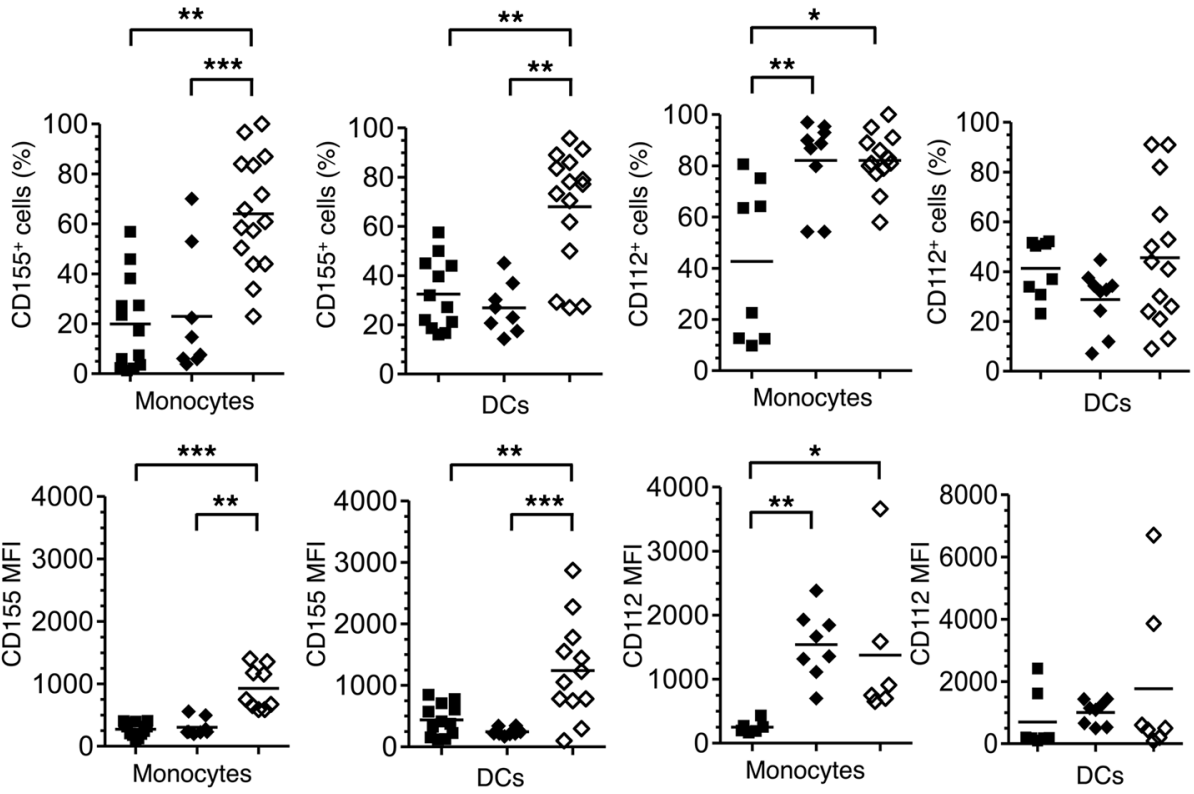

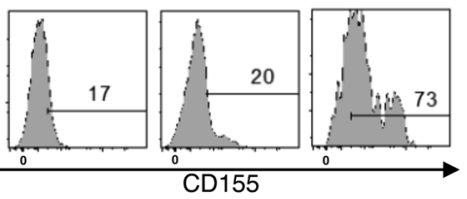

CD3-CD11c+CD14- DCs
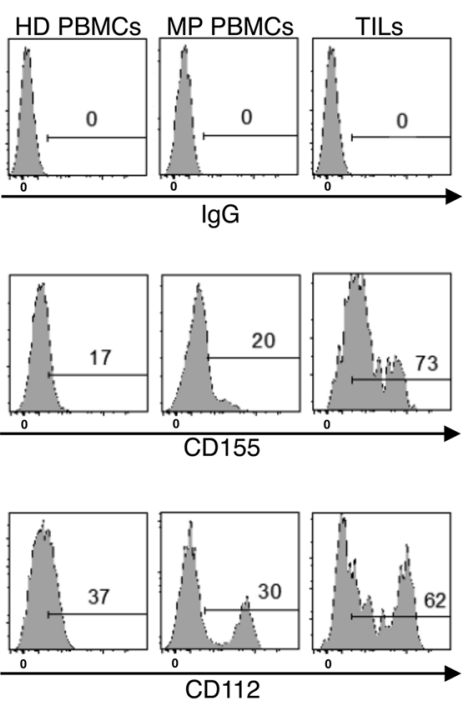

$\diamond$ Melanoma single-cell suspension

Figure 6. The TIGIT ligands CD155 and CD112 are upregulated on metastatic melanoma cells and APCs in the TME. (A) Frequencies of APCs and tumor cells within the $\mathrm{CD}^{-}$cells isolated from metastatic melanoma single-cell suspensions $(n=11)$. (B) Representative flow cytometric analysis of CD155 and CD112

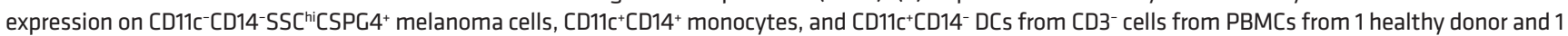
melanoma patient and from 1 metastatic melanoma single-cell suspension. (C) Pooled data showing CD155 ( $n=16)$, CD112 ( $n=10)$, PD-L1 ( $n=20)$, HVEM ( $n=17)$, and galectin-9 $(n=5)$ expression by CSPG4+ cells from melanoma single-cell suspensions. $P$ values were obtained by Friedman's, test followed by Dunn's multiple comparisons test. (D) Pooled data showing CD155 and CD112 expression (percentage and MFI) on monocytes and DCs from PBMCs from healthy donors $(n=12)$ and melanoma patients $(n=8)$ and from metastatic melanoma single-cell suspensions $(n=15)$. $P$ values were obtained by a 1 -way ANOVA, followed by Tukey's multiple comparisons test (bottom row, second panel from the left) and by a Kruskal-Wallis test, followed by Dunn's multiple comparisons test (all other panels). Data are representative of 3 independent experiments. Horizontal bars depict the mean. ${ }^{*} P<0.05 ;{ }^{*} P<0.01 ;{ }^{* *} P<0.001$.

trast, Flu-specific, CMV-specific, total effector, and effector memory $\mathrm{CD}^{+} \mathrm{T}$ cells isolated from PBMCs from the same patients had much lower TIGIT expression levels in terms of percentages and MFI. Strikingly, the majority of TA-specific $\mathrm{CD}^{+} \mathrm{T}$ cells in PBMCs and tumors from patients with advanced melanoma coexpressed TIGIT and PD-1. Our findings are in line with the recent report of elevated TIGIT expression by $\mathrm{CD}^{+}$TILs in a number of mouse and human solid tumors, including lung, colon, breast, uterine, and renal cancers using gene expression analysis (40). In this study, TIGIT was most often coexpressed with PD-1 on the surface of CD $8^{+}$TILs in mouse tumors and in 3 human non-small-cell lung and colon cancer samples. Our findings show that TIGIT ${ }^{+} \mathrm{PD}-1^{+}$ TA-specific $\mathrm{CD}^{+} \mathrm{T}$ cells display an effector memory phenotype and are more differentiated and more activated than TIGIT $^{+} \mathrm{PD}-\mathrm{1}^{-}$ 
A
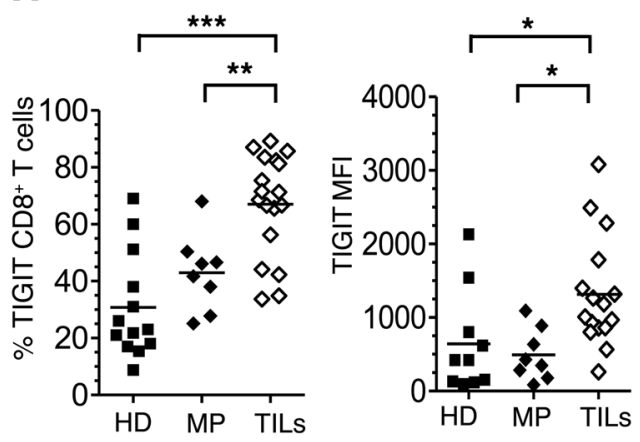

B
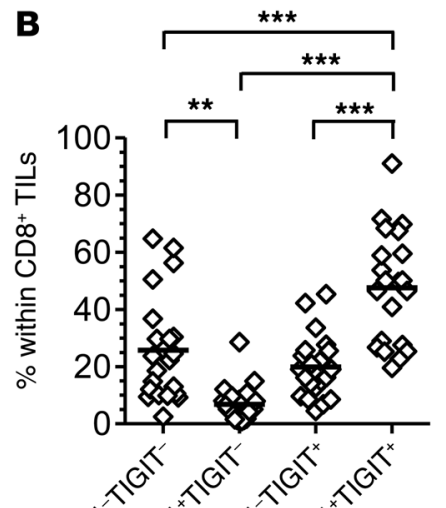
60
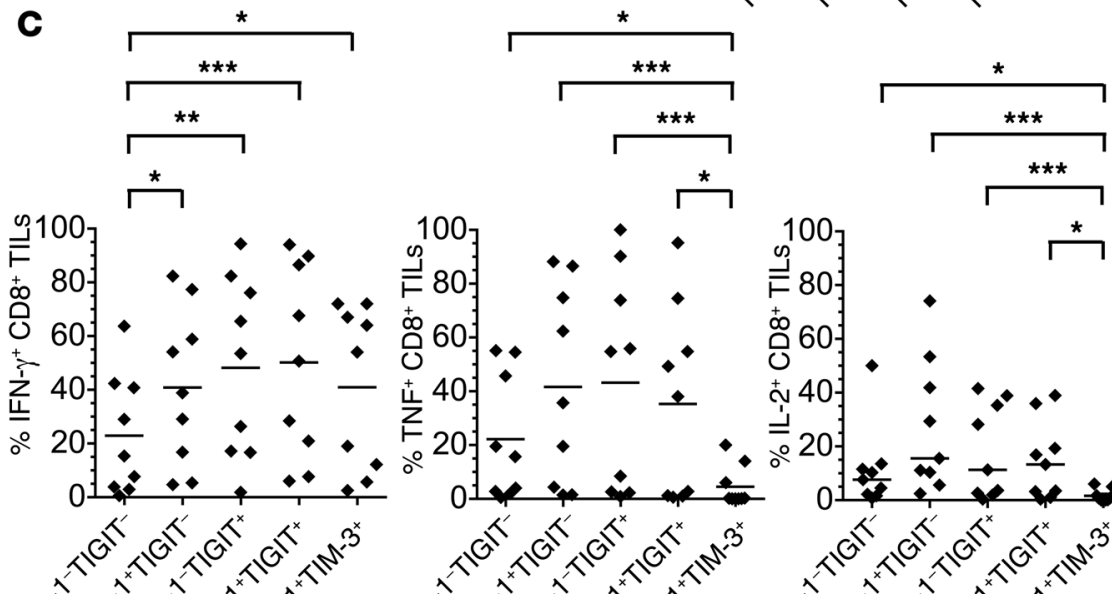

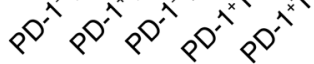
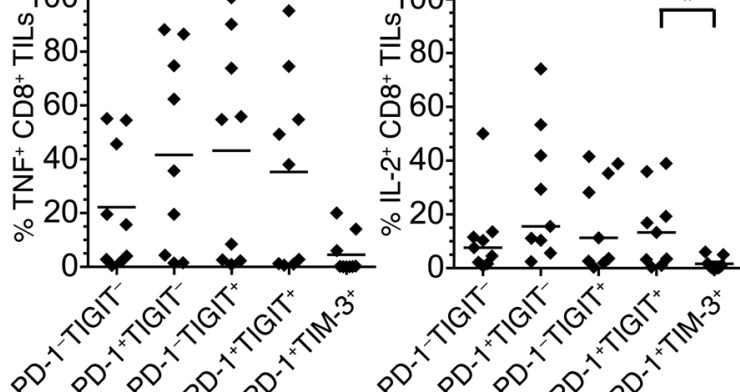

D
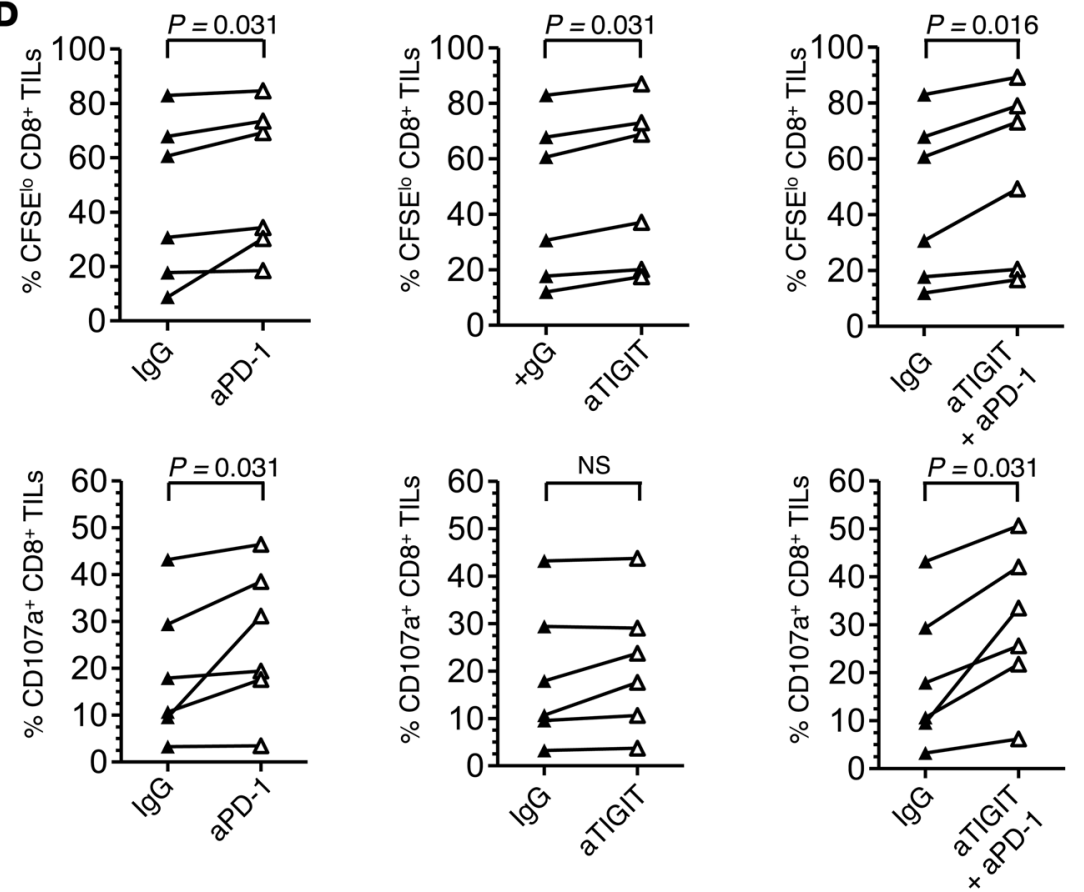

Figure 7. CD8+ TILs upregulate TIGIT and PD-1 and exhibit enhanced proliferation and degranulation upon dual TIGIT and PD-1 blockade. (A) Pooled data showing TIGIT expression (percentage and $\mathrm{MFI}$ ) by $\mathrm{CD}^{+} \mathrm{T}$ cells from PBMCs from healthy donors (HD) $(n=8)$, melanoma patients (MP) $(n=13)$, and melanoma TILs (TILs) $(n=18)$. $P$ values were obtained by a 1-way ANOVA, followed by Tukey's multiple comparisons test (left panel) and a Kruskal-Wallis test, followed by Dunn's multiple comparisons test (right panel). (B) Pooled data showing the frequencies of melanoma $\mathrm{CD}^{+}$TILs ( $n=22$ ) expressing PD-1 and/or TIGIT. $P$ values were obtained by repeated-measures ANOVA, followed by Tukey's multiple comparisons test. (C) Pooled data showing the frequencies of CD8 ${ }^{+}$TILs producing IFN- $\gamma$, TNF, or IL-2 according to PD- 1 and TIGIT expression or PD-1 and TIM-3 coexpression $(n=9)$ after a 6-hour IVS with PMA and ionomycin. $P$ values were obtained by repeated-measures ANOVA, followed by Tukey's (left panel) and Friedman's tests, followed by Dunn's multiple comparisons test (center and right panels). (D) Pooled data showing the percentages of CFSE ${ }^{\text {lo }}$ and CD107a+ CD8 ${ }^{+}$TILs from melanoma patients $(n=6)$ after a 5-day IVS with IFN- $\gamma$-treated autologous $\mathrm{CD}^{-}$cells and anti-CD3 Ab. $P$ values were obtained by Wilcoxon's test. ${ }^{*} P<0.05$;

${ }^{* *} P<0.01$; ${ }^{* *} P<0.001$. Horizontal bars depict the mean. Data are representative of 3 independent experiments.

We provide several lines of evidence supporting the notion that TIGIT is an early $\mathrm{T}$ cell activation marker that is further upregulated by dysfunctional TA-specific $\mathrm{CD} 8^{+} \mathrm{T}$ cells upon chronic stimulation. First, in agreement with previous studies in mice and healthy donors $(16,35-37)$, TA-specific $\mathrm{CD} 8^{+} \mathrm{T}$ cells upregulated TIGIT expression after 1 day of IVS with cognate antigen, while PD-1 upregulation has been shown to occur only after 4 days (5). Second, TA-specific $\mathrm{CD} 8^{+} \mathrm{T}$ cells upregulated TIGIT expression upon PD-1 blockade in the presence of cognate antigen. Finally, highly dysfunctional $\mathrm{PD}-1^{+} \mathrm{TIM}^{-} 3^{+}$TA-specific $\mathrm{CD} 8^{+}$ $\mathrm{T}$ cells $(6,41)$ expressed higher TIGIT levels than did PD-1 ${ }^{+}{ }^{-}{ }^{-1 M}-3^{-}$and PD- ${ }^{-1}{ }^{-T I M}-3^{-}$ TA-specific $\mathrm{CD} 8^{+} \mathrm{T}$ cells.

Unlike the NY-ESO-1-specific CD8 ${ }^{+}$ $\mathrm{T}$ cells that were present in the PBMCs of patients with advanced melanoma, a significant number of $\mathrm{CD} 8^{+}$TILs upregulated TIGIT without PD-1. TIGIT ${ }^{+} \mathrm{PD}-1^{+} \mathrm{CD} 8^{+}$TILs exhibited cytokine-producing frequencies similar to those of TIGIT $^{+} \mathrm{PD}-1^{-}$and TIGIT $^{-}$

and TIGIT-PD- $1^{+} \mathrm{CD} 8^{+} \mathrm{T}$ cell subsets. However, there was no significant difference in activation or differentiation between TIGIT $^{-}$ PD $-1^{+}$and TIGIT $^{+} \mathrm{PD}-1^{-} \mathrm{CD} 8^{+}$TILs, although those subsets were more differentiated and activated than TIGIT-PD-1 $1^{-} \mathrm{CD}^{+}$TILs.
PD $-1^{+} \mathrm{CD}^{+}$TILs, suggesting that the upregulation of TIGIT alone or in combination with $\mathrm{PD}-1$ is not a marker of $\mathrm{CD}^{+} \mathrm{T}$ cell dysfunction. Notably, $\mathrm{TIGIT}^{+} \mathrm{PD}-1^{+}$, $\mathrm{TIGIT}^{+} \mathrm{PD}-1^{-}$, and TIGIT-PD-1+ ${ }^{+} \mathrm{CD}^{+}$ TILs appeared to follow a bimodal distribution with regard to cyto- 
A

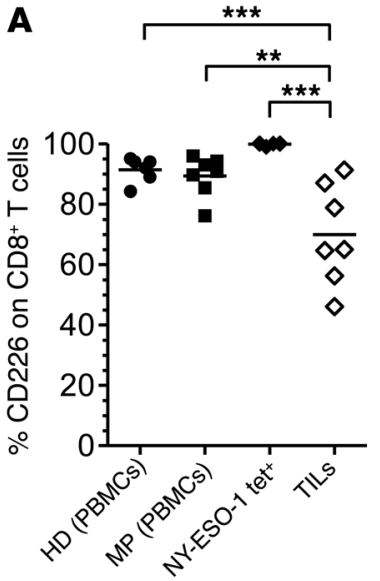

B
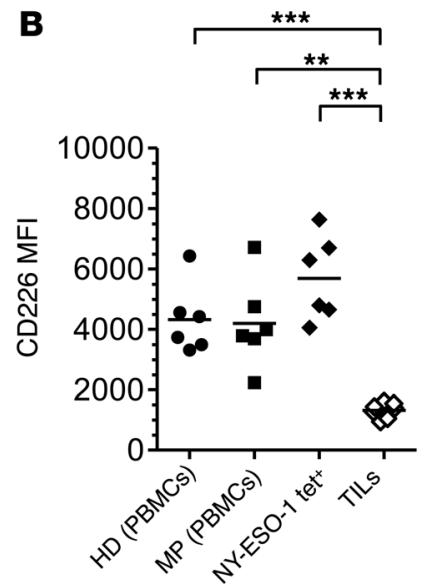

Figure 8. CD8+ ${ }^{+}$TILs downregulate CD226 expression in metastatic melanoma. (A and $\mathbf{B}$ ) Pooled data comparing the frequencies (A) and MFI (B) of CD226 expression by CD8 ${ }^{+}$T cells isolated from PBMCs from healthy donors $(n=6)$ and melanoma patients $(n=6)$, by circulating NY-ESO-1-specific CD8 ${ }^{+}$T cells from melanoma patients $(n=6)$, and by CD8 ${ }^{+}$TILs $(n=7)$ isolated from metastatic melanoma. ${ }^{* *} P<0.01$ and ${ }^{* * *} P<0.001$ by Kruskal-Wallis test, followed by Dunn's multiple comparisons test. Data are representative of 3 independent experiments.

kine production (i.e., exhibiting either low or high cytokine-producing frequencies). This distribution was likely due to the presence of $\mathrm{CD} 8^{+} \mathrm{TIL}$ subsets exhibiting variable levels of $\mathrm{T}$ cell dysfunction, illustrating the functional heterogeneity of PD $-1^{+} \mathrm{CD} 8^{+}$TILs. In this regard, we have previously shown that $\mathrm{TA}$-specific $\mathrm{CD} 8^{+} \mathrm{T}$ cells often coexpress multiple inhibitory receptors including PD- 1 and TIM-3 $(6,7)$. PD-1+TIM-3 ${ }^{+}$TA-specific CD8 ${ }^{+}$cells, which represent a subset of $\mathrm{PD}-1^{+} \mathrm{TA}$-specific $\mathrm{CD} 8^{+} \mathrm{T}$ cells, exhibit higher dysfunctional capacities than do PD-1+ $1^{+} \mathrm{TIM}-3^{-} \mathrm{TA}-$ specific $\mathrm{CD} 8^{+} \mathrm{T}$ cells. In the present study, we show that PD $-1^{+} \mathrm{TIM}-3^{+} \mathrm{CD} 8^{+}$TILs are more dysfunctional in terms of TNF and IL-2 production than are TIGIT $^{+}$PD-1 $1^{+}$, TIGIT $^{+}$PD- $-1^{-}$, or TIGIT-PD-1 $1^{+}$CD $8^{+}$TILs, supporting the presence of multiple $\mathrm{T}$ cell subsets exhibiting variable levels of dysfunction among TIGIT $^{+}$CD8 $8^{+}$TILs.

TIGIT appears to exhibit T cell immunosuppressive effects through multiple mechanisms by (a) enhancing IL-10 production by DCs through CD155 (16), (b) exerting T cell-intrinsic inhibitory effects and/or competing with CD112 for CD155 binding, and/or (c) disrupting CD226 homodimerization (35-37). Our findings suggest that the negative immunoregulatory effects of the TIGIT pathway on human TA-specific $\mathrm{CD} 8^{+} \mathrm{T}$ cells are not mediated through IL-10 production by APCs but rather through direct $\mathrm{T}$ cell-intrinsic effects.

While TIGIT blockade alone augments TA-specific CD8 ${ }^{+} \mathrm{T}$ cell proliferation and cytokine production, dual TIGIT and PD-1 blockade further increases the percentages of total TA-specific, cytokine-producing, and proliferating $\mathrm{CD} 8^{+} \mathrm{T}$ cells. In addition, upon TCR stimulation, dual TIGIT/PD-1 blockade was superior to TIGIT blockade alone in augmenting the proliferation and degranulation of $\mathrm{CD}^{+}$TILs isolated from metastatic melanoma. Our findings in patients with advanced melanoma are in line with data from a number of experiments in mice showing the role of PD-1 and TIGIT blockade in inducing tumor regression (40 and

Korman et al., unpublished observations). Strikingly, in sharp contrast to the flow cytometric data for CT26 mouse tumors and the gene expression analysis of squamous cell lung cancers showing high expression levels of both TIGIT and CD226 at tumor sites (40), $\mathrm{CD}^{+}$TILs in metastatic melanoma expressed low levels of CD226, while TA-specific CD8 ${ }^{+} \mathrm{T}$ cells upregulated CD226 in the periphery. These findings indicate an imbalance of TIGIT/ CD226 expression by CD8 ${ }^{+}$TILs in metastatic melanoma that may explain the modest effect of TIGIT blockade on the proliferation and function of $\mathrm{CD} 8^{+}$TILs as compared with circulating $\mathrm{TA}$-specific $\mathrm{CD} 8^{+} \mathrm{T}$ cells.

Within metastatic melanoma tumors, the expression of CD155 and CD112 by monocytes and DCs was substantially higher than in PBMCs from the same melanoma patients and in PBMCs from healthy donors. Interestingly, the large majority of melanoma cells isolated from metastatic melanoma expressed CD155 and CD112 ex vivo. These findings are in line with 1 immunostaining study showing that CD155 is expressed by melanomas and correlates with melanoma progression (31). In addition, the frequencies of melanoma cells expressing CD155 and CD122 were higher than of those expressing PD-L1, HVEM, and galectin-9, suggesting that the interaction of CD155 and CD112 with their ligands plays an important role in regulating TA-specific $\mathrm{T}$ cell responses at tumor sites. Although PD-L1 expression was clearly induced by inflammatory cytokines including IFN- $\gamma$, we observed no upregulation of CD155 or CD112 by melanoma cells upon exposure to IFN- $\gamma$ or TNF (Chauvin et al., unpublished observations) and found no correlation between the percentages of PD-L1+ melanoma cells and the percentages of $\mathrm{CD} 155^{+}$or $\mathrm{CD} 122^{+}$melanoma cells ex vivo, suggesting that CD155 and CD112 upregulation by melanoma cells is supported by mechanisms other than PD-L1 expression.

In summary, the findings in this study demonstrate that TIGIT is coexpressed with PD-1 by the large majority of TA-specific $\mathrm{CD}^{+} \mathrm{T}$ cells in the periphery and at tumor sites in patients with advanced melanoma. In addition, CD226 was downregulated by CD8 ${ }^{+}$TILs, while the TIGIT ligands CD155 and CD112 were upregulated by the majority of melanoma cells, monocytes, and DCs in metastatic melanoma, suggesting that the TIGIT pathway plays a major immunoregulatory role in metastatic melanoma. Importantly, TIGIT blockade adds to PD-1 blockade to enhance TA-specific $\mathrm{CD} 8^{+} \mathrm{T}$ cell function and proliferation in the periphery and at tumor sites. Therefore, the present findings strongly support the use of dual TIGIT and PD-1 blockade to stimulate potent antitumor $\mathrm{CD}^{+} \mathrm{T}$ cell responses in patients with advanced melanoma.

\section{Methods}

TA-specific $C D 8^{+} T$ cell phenotypic analysis. Using MACS Column Technology (Miltenyi Biotec), CD8 ${ }^{+} \mathrm{T}$ lymphocytes from PBMCs obtained from patients were purified to greater than $95 \%$ and incubated for 30 minutes at room temperature with APC-labeled HLA-A2/NY-ESO-1 157-165, HLA-A2/CMV 495-503, HLA-A2/Flu-M 58-66, or (as a negative control) HLA-A2/HIVpol 476-484 tetramers (TCMetrix). The minimum percentage of antigen-specific $\mathrm{CD}^{+} \mathrm{T}$ cells detected in patients using these tetramers was $0.01 \%$ of total $\mathrm{CD} 8^{+} \mathrm{T}$ cells. Next, for membrane staining, cells were incubated for 20 minutes at $4^{\circ} \mathrm{C}$ with a combination of the following conjugated $\mathrm{Abs}$ and reagents: CD8-V500, CD38-PerCp-Cy5.5, and CD69-FITC (from BD); 
CD8-PeCy-7, CD45RO-ECD, HLA-DR-ECD, and CD57-FITC (from Beckman Coulter); PD-1-PeCy7 and CD226-PE (from BioLegend); CD45RA-PerCP-Cy5.5 and TIGIT-PE or TIGIT-PerCP-efluor710 (from eBioscience); and TIM-3-PE and CCR7-FITC (from R\&D Systems). A violet amine-reactive dye (Invitrogen) was used to assess the viability of the cells. At least 2 million events were collected during flow cytometric analysis using a FACSAria flow cytometer (BD) and analyzed using FlowJo software (Tree Star Inc.).

Generation of anti-TIGIT Abs. Anti-human TIGIT Abs were generated in HuMab mice $(42,43)$ immunized with a TIGIT-Fc fusion protein. Abs, which bound to full-length TIGIT expressed on CHO transfectants, were tested for their ability to block the binding of TIGIT-Fc to CHO transfectants expressing CD155 (PVR). The anti-TIGIT mAb 10D7.G8 (IgG4) was selected and found to bind well to activated human T cells. 10D7.G8 was also shown to enhance NF- $\kappa B$ reporter gene expression in full-length TIGIT transfectants of Jurkat cells incubated with $\mathrm{CHO}$ cells expressing membrane-bound, single-chain antiCD3 and full-length human PVR.

CFSE proliferation assay. For PBMCs from melanoma patients, 4.5 million CFSE-labeled cells per condition and per well of a 24-well plate were incubated for 6 days in 10\% human serum Iscove's DMEM containing $50 \mathrm{IU} / \mathrm{ml}$ recombinant human IL-2 (rhIL-2) (PeproTech) with NY-ESO-1 157-165 or HIVpol 476-484 $(2 \mu \mathrm{g} / \mathrm{ml})$ peptide in the presence of $10 \mu \mathrm{g} / \mathrm{ml}$ fully human anti-PD-1 (BMS-936558, IgG4, BMS) and/or anti-TIGIT (10D7.G8, IgG4, BMS) blocking mAbs or IgG4 isotype control Ab (DT-1D12-g4P, BMS). On day 6, cells were stained with APC-labeled HLA-A2/NY-ESO-1 157-165 tetramers, then CD14-APC-Cy7, CD19-APC-Cy7 (BD), CD8-PerCP-Cy5.5 (BioLegend), TIGIT-PE, (eBioscience), and PD-1-PeCy7 (Beckman Coulter), followed by incubation with a violet anime-reactive dye. Two million events were collected during flow cytometric analysis using a FACSAria flow cytometer. CD8 ${ }^{+}$TILs and non-CD3 cells were isolated from tumor single-cell suspensions using MACS Column Technology. CD8 ${ }^{+}$TILs were rested for 2 days in Iscove's DMEM and $100 \mathrm{IU} / \mathrm{ml} \mathrm{rhIL}-2$, while non-CD3 cells were treated with $100 \mathrm{ng} / \mathrm{ml}$ IFN- $\gamma$. CD 8 cells were labeled with CFSE and coincubated with non -CD3 for 5 days at a 1:1 ratio in the presence or absence of IgG, antiPD-1, and/or anti-TIGIT mAbs in medium containing $100 \mathrm{IU} / \mathrm{ml}$ rhIL-2 and $0.1 \mu \mathrm{g} / \mathrm{ml} \mathrm{OKT3} \mathrm{(eBioscience)} \mathrm{to} \mathrm{provide} \mathrm{stimulation}$ signals. On day 5, cells were stained with CD14-ECD, CD4-PeCy-7 (Beckman Coulter), CD19 Pacific Blue, CD107a-PerCP-Cy5.5, and CD8-APC (BD), followed by incubation with a violet anime-reactive dye. Flow cytometry was performed using an LSR-II flow cytometer (BD) and the data analyzed using FlowJo software.

Intracellular cytokine staining assay. For PBMCs from melanoma patients, 4.5 million cells were incubated for 6 days in Iscove's DMEM containing $50 \mathrm{IU} / \mathrm{ml}$ rhIL-2 with NY-ESO-1 157-165 or HIVpol 476-484 $(2 \mu \mathrm{g} / \mathrm{ml})$ peptide in the presence of anti-PD-1- and/or anti-TIGITblocking mAbs, isotype control Abs, or TIGIT-Fc fusion protein. On day 6, cells were restimulated for 6 hours with NY-ESO-1 157-165 or HIVpol 476-484 peptide as a control $(10 \mu \mathrm{g} / \mathrm{ml})$. After 1 hour, brefeldin A (Sigma-Aldrich) was added to the culture medium $(10 \mu \mathrm{g} / \mathrm{ml})$. For TIL ex vivo analysis, CD8 ${ }^{+}$TILs were isolated from tumor single-cell suspensions using MACS Column Technology and then stimulated for 6 hours with $1 \mu \mathrm{g} / \mathrm{ml}$ PMA and $2.5 \mu \mathrm{g} / \mathrm{ml}$ ionomycin (Sigma-Aldrich). Brefeldin A was added to the medium after 1 hour of stimulation. Cells were then stained with APC-labeled HLA-A2/NY-ESO-1 157-165 tetramers (for PBMC analysis only), then CD4-PeCy7-, CD14-ECD-, CD19-ECD- (Beckman Coulter), and CD8-V500-conjugated (BD) $\mathrm{Abs}$, and then incubated with a violet anime-reactive dye. Intracellular staining was performed with IFN- $\gamma$-FITC (Miltenyi Biotec), TNFAlexa Fluor 700 (BD), and IL-2-PerCp-Cy5.5 (BioLegend) Abs. Two million events were collected during flow cytometric analysis on a FACSAria flow cytometer for PBMC analysis, and 0.1 million events were collected using an LSR-II flow cytometer for TIL analysis.

IL-10 detection. PBMCs (4.5 million) from patients with advanced melanoma were incubated for 6 days in Iscove's DMEM containing 50 IU/ml rhIL-2 and stimulated with NY-ESO-1 157-165 or HIVpol 476$484(2 \mu \mathrm{g} / \mathrm{ml})$ peptide in the presence of $10 \mu \mathrm{g} / \mathrm{ml}$ anti-PD-1- and/ or anti-TIGIT-blocking mAbs. Supernatants were collected for IL-10 detection after 6 days of incubation. The concentration of IL-10 in supernatants was determined using a BD OptEIA Human IL-10 ELISA Set (BD Biosciences).

Ex vivo phenotypic analysis of subsets from tumor single-cell suspensions. Cells from tumor single-cell suspensions were divided and placed into several tubes for analysis. Tumor cells were detected using a mouse anti-human CSPG4 Ab (763.74; gift of S. Ferrone, Massachusetts General Hospital, Boston, Massachusetts, USA) targeted with FITC goat anti-mouse Abs (Beckman Coulter). Cells were washed with PBS-based buffer containing mouse serum, then labeled with CD155-PE (eBioscience) or CD112-PE (BioLegend) and a mix of CD3-PerCPCy5.5 (BioLegend), CD19-APC-Cy7 (BD), CD14-ECD, CD56-PeCy7 (Beckman Coulter), and CD11c Alexa Fluor 700 (eBioscience) Abs. In another tube, cells were labeled with CD226-biotin (Abcam), then streptavidin-ECD (Invitrogen), TIGIT-PE, CD11c Alexa Fluor 700 (eBioscience), CD56-FITC (Beckman Coulter), CD4-PerCPCy5.5 (BioLegend), CD14-APC-Cy7, CD19-APC-Cy7, and CD8-V500 (BD). All samples were also incubated with the violet anime-reactive dye to exclude dead cells. Flow cytometry was performed using an LSR-II or a Fortessa (BD) flow cytometer, and data analysis was performed using FlowJo software. MFI was compared between experiments analyzed on the LSR-II flow cytometer.

Statistics. The normality of each variable was evaluated using the Shapiro-Wilk test. In cases of normally distributed data, the comparison of variables was performed using a 1-way ANOVA or a repeated-measures ANOVA for unpaired and paired data, respectively, followed by a Tukey's multiple comparisons test. When the data were not normally distributed, the comparison of variables was performed with a Kruskal-Wallis test or a Friedman test for unpaired and paired data, respectively, followed by a Dunn's multiple comparisons test. In these tests, $P$ values of less than 0.05 were considered significant. These analyses were performed with SAS software, version 9.1 (SAS Institute), and GraphPad Prism 6 (GraphPad Software).

Study approval. Blood samples were obtained from 11 healthy volunteers or from 11 HLA-A2 $2^{+}$patients with NY-ESO-1-expressing stage IV melanoma under the University of Pittsburgh Cancer Institute's IRB-approved protocols 00-079, 05-140, or 96-099. NY-ESO-1 expression in tumors from melanoma patients was assessed by RT-PCR and IHC. Serum NY-ESO-1-specific Abs from all selected patients were detected by ELISA. PBMCs from 9 patients who exhibited responses against NY-ESO-1 157-165 were used. NY-ESO-1-specific $\mathrm{CD}^{+} \mathrm{T}$ cells were detected by flow cytometry using APC-labeled HLA-A2/NY-ESO-1 157-165 tetramers. The percentages of detectable NY-ESO-1 157-165-specific CD8 ${ }^{+}$T cells isolated from patients' 
PBMCs ranged from $0.01 \%$ to $5.7 \%$ of total $\mathrm{CD} 8^{+} \mathrm{T}$ cells (median, $0.03 \%$ ). Tumor and TIL samples were collected under the University of Pittsburgh Cancer Institute's IRB-approved protocol 96-099.

\section{Acknowledgments}

This work was supported by National Cancer Institute (NCI), NIH grants R01CA112198 and R01CA157467 (to H.M. Zarour) and
P50CA121973 (to J.M. Kirkwood), and by a research grant from Bristol-Myers Squibb (to H.M. Zarour).

Address correspondence to: Hassane M. Zarour, Hillman Cancer Center, Research Pavilion, Suite 1.32a, 5117 Centre Avenue, Pittsburgh, Pennsylvania 15213-2582, USA. Phone: 412.623.3272; E-mail: zarourhm@upmc.edu.
1. Boon T, Coulie PG, Van den Eynde BJ, van der Bruggen P. Human T cell responses against melanoma. Annu Rev Immunol. 2006;24:175-208.

2. Wherry EJ, Barber DL, Kaech SM, Blattman JN, Ahmed R. Antigen-independent memory CD8 T cells do not develop during chronic viral infection. Proc Natl Acad Sci U S A. 2004;101(45):16004-16009.

3. Wherry EJ. T cell exhaustion. Nat Immunol. 2011;12(6):492-499.

4. Ahmadzadeh $\mathrm{M}$, et al. Tumor antigen-specific CD8 T cells infiltrating the tumor express high levels of PD-1 and are functionally impaired. Blood. 2009;114(8):1537-1544.

5 . Fourcade J, et al. PD-1 is a regulator of NY-ESO1-specific CD8 ${ }^{+} \mathrm{T}$ cell expansion in melanoma patients. JImmunol. 2009;182(9):5240-5249.

6. Fourcade J, et al. Upregulation of Tim-3 and PD-1 expression is associated with tumor antigen-specific $\mathrm{CD}^{+} \mathrm{T}$ cell dysfunction in melanoma patients. J Exp Med. 2010;207(10):2175-2186.

7. Fourcade J, et al. CD8(+) T cells specific for tumor antigens can be rendered dysfunctional by the tumor microenvironment through upregulation of the inhibitory receptors BTLA and PD-1. Cancer Res. 2012;72(4):887-896.

8. Sakuishi K, Apetoh L, Sullivan JM, Blazar BR, Kuchroo VK, Anderson AC. Targeting Tim-3 and PD-1 pathways to reverse T cell exhaustion and restore anti-tumor immunity. J Exp Med. 2010;207(10):2187-2194.

9. Barber DL, et al. Restoring function in exhausted CD8 T cells during chronic viral infection. Nature. 2006;439(7077):682-687.

10. Blackburn SD, et al. Coregulation of $\mathrm{CD}^{+} \mathrm{T}$ cell exhaustion by multiple inhibitory receptors during chronic viral infection. Nat Immunol. 2009;10(1):29-37.

11. Topalian SL, et al. Survival, durable tumor remission, and long-term safety in patients with advanced melanoma receiving nivolumab. J Clin Oncol. 2014;32(10):1020-1030.

12. Hamid $O$, et al. Safety and tumor responses with lambrolizumab (anti-PD-1) in melanoma. N Engl JMed. 2013;369(2):134-144.

13. Wolchok JD, et al. Nivolumab plus ipilimumab in advanced melanoma. N Engl JMed. 2013;369(2):122-133.

14. Boles KS, et al. A novel molecular interaction for the adhesion of follicular CD $4 \mathrm{~T}$ cells to follicular DC. Eur J Immunol. 2009;39(3):695-703.

15. Stengel KF, et al. Structure of TIGIT immunoreceptor bound to poliovirus receptor reveals a cell-cell adhesion and signaling mechanism that requires cis-trans receptor clustering. Proc Natl Acad Sci U S A. 2012;109(14):5399-5404.
16. Yu X, et al. The surface protein TIGIT suppresses $T$ cell activation by promoting the generation of mature immunoregulatory dendritic cells. Nat Immunol. 2009;10(1):48-57.

17. Stanietsky N, et al. The interaction of TIGIT with PVR and PVRL2 inhibits human NK cell cytotoxicity. Proc Natl Acad Sci U S A. 2009;106(42):17858-17863.

18. Shibuya A, et al. DNAM-1, a novel adhesion molecule involved in the cytolytic function of $\mathrm{T}$ lymphocytes. Immunity. 1996;4(6):573-581.

19. Shibuya K, et al. CD226 (DNAM-1) is involved in lymphocyte function-associated antigen 1 costimulatory signal for naive $T$ cell differentiation and proliferation. JExp Med. 2003;198(12):1829-1839.

20. Fuchs A, Cella M, Giurisato E, Shaw AS, Colonna M. Cutting edge: CD96 (tactile) promotes NK cell-target cell adhesion by interacting with the poliovirus receptor (CD155). JImmunol. 2004;172(7):3994-3998.

21. Bottino C, et al. Identification of PVR (CD155) and Nectin-2 (CD112) as cell surface ligands for the human DNAM-1 (CD226) activating molecule. JExp Med. 2003;198(4):557-567.

22. Tahara-Hanaoka S, et al. Functional characterization of DNAM-1 (CD226) interaction with its ligands PVR (CD155) and nectin-2 (PRR-2/CD112). Int Immunol. 2004;16(4):533-538.

23. Mendelsohn CL, Wimmer E, Racaniello VR. Cellular receptor for poliovirus: molecular cloning, nucleotide sequence, and expression of a new member of the immunoglobulin superfamily. Cell. 1989;56(5):855-865.

24. Freistadt MS, Fleit HB, Wimmer E. Poliovirus receptor on human blood cells: a possible extraneural site of poliovirus replication. Virology. 1993;195(2):798-803.

25. Kamran N, Takai Y, Miyoshi J, Biswas SK, Wong JS, Gasser S. Toll-like receptor ligands induce expression of the costimulatory molecule CD155 on antigen-presenting cells. PLoS One. 2013;8(1):e54406.

26. Reymond N, et al. DNAM-1 and PVR regulate monocyte migration through endothelial junctions. J Exp Med. 2004;199(10):1331-1341.

27. Tucker SP, Thornton CL, Wimmer E, Compans RW. Bidirectional entry of poliovirus into polarized epithelial cells. J Virol. 1993;67(1):29-38.

28. Lopez M, Aoubala M, Jordier F, Isnardon D, Gomez S, Dubreuil P. The human poliovirus receptor related 2 protein is a new hematopoietic/endothelial homophilic adhesion molecule. Blood. 1998;92(12):4602-4611.

29. Pende D, et al. Expression of the DNAM-1 ligands, Nectin-2 (CD112) and poliovirus recep- tor (CD155), on dendritic cells: relevance for natural killer-dendritic cell interaction. Blood. 2006;107(5):2030-2036.

30. Ardolino M, et al. DNAM-1 ligand expression on Ag-stimulated T lymphocytes is mediated by ROS-dependent activation of DNA-damage response: relevance for NK-T cell interaction. Blood. 2011;117(18):4778-4786.

31. Bevelacqua V, et al. Nectin like-5 overexpression correlates with the malignant phenotype in cutaneous melanoma. Oncotarget. 2012;3(8):882-892.

32. Casado JG, et al. Expression of adhesion molecules and ligands for activating and costimulatory receptors involved in cell-mediated cytotoxicity in a large panel of human melanoma cell lines. Cancer Immunol Immunother. 2009;58(9):1517-1526.

33. Oshima T, et al. Nectin-2 is a potential target for antibody therapy of breast and ovarian cancers. Mol Cancer. 2013;12:60.

34. Sloan KE, et al. CD155/PVR plays a key role in cell motility during tumor cell invasion and migration. BMC Cancer. 2004;4:73.

35. Joller N, et al. Cutting edge: TIGIT has T cell-intrinsic inhibitory functions. JImmunol. 2011;186(3):1338-1342.

36. Levin SD, et al. Vstm 3 is a member of the CD28 family and an important modulator of T-cell function. Eur J Immunol. 2011;41(4):902-915.

37. Lozano E, Dominguez-Villar M, Kuchroo V, Hafler DA. The TIGIT/CD226 axis regulates human T cell function. JImmunol. 2012;188(8):3869-3875.

38. Zhang Y, et al. Genome-wide DNA methylation analysis identifies hypomethylated genes regulated by FOXP3 in human regulatory T cells. Blood. 2013;122(16):2823-2836.

39. Joller N, et al. Treg cells expressing the coinhibitory molecule TIGIT selectively inhibit proinflammatory Th1 and Th17 cell responses. Immunity. 2014;40(4):569-581.

40. Johnston RJ, et al. The immunoreceptor TIGIT regulates antitumor and antiviral CD8(+) T cell effector function. Cancer Cell. 2014; 26(6):923-937.

41. Fourcade J, et al. PD-1 and Tim-3 regulate the expansion of tumor antigen-specific CD8(+) T cells induced by melanoma vaccines. Cancer Res. 2014;74(4):1045-1055.

42. Lonberg N, et al. Antigen-specific human antibodies from mice comprising four distinct genetic modifications. Nature. 1994;368(6474):856-859.

43. Fishwild DM, et al. High-avidity human IgGк monoclonal antibodies from a novel strain of minilocus transgenic mice. Nat Biotechnol. 1996;14(7):845-851. 